\title{
Point-stationarity in $d$ dimensions and Palm theory
}

\author{
HERMANN THORISSON \\ Science Institute, University of Iceland, Dunhaga 3, 107 Reykjavik, Iceland. \\ E-mail:hermann@hi.is
}

This paper extends to $d>1$ dimensions the concept of point-stationarity, which formalizes the intuitive idea of a point process for which the behaviour relative to a given point of the process is independent of the point selected as origin. After defining point-stationarity, this concept is characterized in several ways and the characterizations then used to extend to $d$ dimensions a particular approach to Palm theory, producing two dualities between stationary and point-stationary processes with quite different interpretations. The dualities coincide in the ergodic case.

Keywords: coupling; Palm theory; point process; random field; stationarity; stochastic geometry

\section{Introduction}

The main purpose of this paper is to extend to $d>1$ dimensions the concept of pointstationarity, which formalizes the intuitive idea of a simple point process for which the behaviour relative to a given point of the process is independent of the point selected as origin.

Note that point-stationarity is different from stationarity. Stationarity means that the behaviour of the point process relative to any given non-random site is independent of the site selected as origin. For instance, when $d=1$, the Poisson process with constant intensity is stationary since if the origin is moved from 0 to any other fixed site $t$ then we still have a Poisson process with the same intensity. But if a point is added at 0 then we obtain a non-stationary process since if the origin is moved from the point which we placed at 0 to any other fixed site $t$ then there need not be a point there. This new process, however, is point-stationary since, seen from the point which we placed at 0 , the intervals between points are i.i.d. exponential and this will still be true if we move the origin, for instance, to the first point on the right of the point at 0 .

The point-stationarity problem is presented in Section 2 and solved in Section 3.

After defining point-stationarity in Section 3 the concept is further characterized in Sections 4, 5 and 6. In Sections 8 and 9 these characterizations are then used to extend to $d>1$ dimensions a particular approach to the Palm theory of stationary point processes presented in Thorisson (1995) for the case $d=1$, while Section 7 contrasts this approach to the conventional one.

Section 10 concludes with comments on the two Palm dualities presented in Sections 8 and 9, on references, and on possible extensions of the point-stationarity concept, for 
instance, to the zero set of Brownian motion discussed by Mandelbrot (1983) and Kagan and Vere-Jones (1988). These are in fact the only references that this author is aware of where the point-stationarity problem is even mentioned.

The paper is fairly self-contained. In particular, no prior knowledge of Palm theory is assumed. Only in the proof of the final two theorems do we refer to Thorisson (1996) for a coupling result and to Daley and Vere-Jones (1988) for an ergodic theorem.

\section{The point-stationarity problem}

This section explains the point-stationarity problem in full detail, moving from the obvious one-dimensional case to the not so obvious higher-dimensional case. Necessary notation is introduced along the way.

\subsection{The simple point processes $N$ and $N^{\mathrm{o}}$}

Intuitively a simple point process in $d$ dimensions, $d \geqslant 1$, is a countable set of isolated points scattered over the $d$-dimensional Euclidean space $\mathbb{R}^{d}$ in some random manner (like planets scattered over space). This random set of points can be represented by a collection of random variables

$$
N=\left(N(B): B \in \mathscr{B}^{d}\right),
$$

where $\mathscr{B}^{d}$ are the Borel subsets of $\mathbb{R}^{d}$ and $N(B)$ is the number of points in the set $B$. More precisely, a simple point process in $d$ dimensions is a random element $N$ in the measurable space $(M, \mathscr{C})$, where $M$ is the set of all simple counting measures (that is, integer-valued measures $\mu$ on $\left(\mathbb{R}^{d}, \mathscr{S}^{d}\right)$ with $\mu(B)<\infty$ for all bounded $B \in \mathscr{S}^{d}$ and $\mu(\{t\})=0$ or 1 for all $t \in \mathbb{R}^{d}$ ) and $\mathscr{C}$ is the product $\sigma$-algebra on $M$ (the smallest $\sigma$-algebra such that, for each $B \in \mathscr{B}^{d}$, the projection mapping from $M$ to $R$ taking $\mu$ to $\mu(B)$ is $\mathscr{C l} / \mathscr{B}$ measurable). The $\sigma$-algebra $\mathscr{C} b$ is also generated by the usual topology on $M$, but we shall not need this fact. is,

We shall write $N^{\mathrm{o}}$ to indicate that one of the points is placed at 0 (the origin of $\mathbb{R}^{d}$ ), that

$$
N^{\mathrm{o}}(\{0\}) \equiv 1
$$

We shall regard $N^{\mathrm{o}}$ as a random element in $\left(M^{\mathrm{o}}, \mathscr{C l}^{\mathrm{o}}\right)$, where $M^{\mathrm{o}}$ is the subset of $M$ containing the simple counting measures having mass one at the origin,

$$
M^{\mathrm{o}}=\{\mu \in M: \mu(\{0\})=1\},
$$

and $\mathscr{C}^{\circ}$ is the trace of $M^{\circ}$ on $\mathscr{C l}$, that is, $\mathscr{C l}^{\circ}=\mathscr{C} \cap M^{\circ}$.

Let $(\Omega, \mathscr{F}, P)$ be a common probability space supporting $N$ and $N^{\mathrm{o}}$, and all other random elements in this paper. (In Sections 8 and 9 we break this convention by letting there be two choices of $P$ ). 


\subsection{Sites and points}

Call a (non-random) element $t$ of $\mathbb{R}^{d}$ a site (and not a point) to distinguish it from the points of the point process. Thus a random element $T$ in $\mathbb{R}^{d}$ is a random site. Call a random site $\Pi$ a point or a random point of $N$ only if the point process $N$ has a point at $\Pi$, that is, only if

$$
N(\{\Pi\}) \equiv 1 \text {. }
$$

Similarly, call a random site $\Pi^{\mathrm{o}}$ a point or a random point of $N^{\mathrm{o}}$ only if the point process $N^{\mathrm{o}}$ has a point at $\Pi^{\mathrm{o}}$, that is, only if

$$
N^{\mathrm{o}}\left(\left\{\Pi^{\mathrm{o}}\right\}\right) \equiv 1
$$

A point process typically does not look the same seen from a non-random observation site as seen from an observation point (the universe does not look the same seen from space as seen from a planet).

\subsection{Site-shifts and stationarity of $N$}

For $\mu \in M$ let $s(\mu)$ denote the set of $\mu$-points (the point pattern or the support of $\mu$ ):

$$
s(\mu)=\left\{p \in \mathbb{R}^{d}: \mu(\{p\})=1\right\},
$$

For $t \in \mathbb{R}^{d}$, define the shift or site-shift $\theta_{t}$ taking $\mu \in M$ to $\theta_{t} \mu \in M$ by

$$
\theta_{t} \mu(B)=\mu(t+B), \quad B \in \mathscr{B}^{d},
$$

where $t+B=\{t+s: s \in B\}$. The point process $N$ is called stationary if

$$
\theta_{t} N \stackrel{D}{=} N, \quad t \in \mathbb{R}^{d}
$$

where ' $\stackrel{D}{=}$ ' denotes identity in distribution. Note that $\theta_{t}$ shifts (or translates) the point pattern by $-t$. In other words, $\theta_{t}$ shifts the origin (the observation site) to $t$. Thus one could say that stationarity of the point process $N$ means that it looks the same from all non-random observation sites.

\subsection{The point-stationarity problem for $N^{\mathrm{o}}$}

Similarly, it would be natural to say that the point process $N^{\mathrm{o}}$ is point-stationary if it looks the same from all observation points. What this means has not been clear, however - except in one dimension.

When $d=1$, point-stationarity means that $N^{\mathrm{o}}$ is interval-stationary, that is, the intervals between the points form a stationary sequence:

$$
\left(\Pi_{n+k}^{0}-\Pi_{n+k-1}^{\mathrm{o}}\right)_{-\infty<k<\infty} \stackrel{D}{=}\left(\Pi_{k}^{\mathrm{o}}-\Pi_{k-1}^{\mathrm{o}}\right)_{-\infty<k<\infty}, \quad n \in \mathbb{Z},
$$

where 


$$
\ldots<\Pi_{-2}^{\mathrm{o}}<\Pi_{-1}^{\mathrm{o}}<\Pi_{0}^{\mathrm{o}}=0<\Pi_{1}^{\mathrm{o}}<\Pi_{2}^{\mathrm{o}}<\ldots
$$

are the points of $N^{\mathrm{o}}$ written as an increasing sequence. This definition of point-stationarity when $d=1$ can be rewritten as

$$
\theta_{\Pi_{n}^{\mathrm{o}}} N^{\mathrm{o}} \stackrel{D}{=} N^{\mathrm{o}}, \quad n \in \mathbb{Z} .
$$

In other words, if the observer moves from the point at the origin to the $n$th point on the right (or left) of the origin then the probability distribution of the point pattern that he sees does not change: the point process $N^{\mathrm{o}}$ looks the same from all observation points.

When $d>1$, this definition of point-stationarity does not work since then there are no intervals between the points of $N^{\mathrm{o}}$ to form a stationary sequence, and the observer cannot use the simple point-selection rule 'move from the point at the origin to the $n$th point on the right (or left)' since there is typically no $n$th point on the right (or left). But is there some similar way of moving between points in higher dimensions?

\subsection{The point-stationarity problem in the Poisson case}

Note that even the Poisson process seems to present a problem. Let $N$ be a stationary Poisson process. When $d=1$ we obtain a point-stationary $N^{\mathrm{o}}$ by adding a point at the origin to $N$,

$$
N^{\mathrm{o}} \equiv N+\delta_{0}
$$

where $\delta_{0}$ is the measure with mass one at 0 . It is well known that the intervals between points are then i.i.d. exponential and point-stationarity follows. But when $d>1$ (in the plane, for instance) how can we shift the origin to another point of $N^{\mathrm{o}}$ without spoiling the distribution of the point pattern that we see?

'Why not shift the origin to the closest point?' tends to be the first reaction to this question. In order to indicate why this does not work, let us consider the following example.

Example 2.1. Consider the case when $d=1$ and let $N^{\mathrm{o}}$ be the Poisson process with a point at the origin defined in (2.1). We know that $N^{\mathrm{o}}$ is point-stationary so if shifting to the closest point is to be the way to shift in higher dimensions it ought to work in one dimension also, that is, it should not change the distribution of $N^{\mathrm{o}}$.

Shift the origin of $N^{\mathrm{o}}$ to the closest point to obtain $\theta_{\Pi_{\text {closest }}^{\mathrm{o}}} N^{\mathrm{o}}$, where

$$
\Pi_{\text {closest }}^{\mathrm{o}}= \begin{cases}\Pi_{1}^{\mathrm{o}} & \text { if } \Pi_{1}^{\mathrm{o}} \leqslant-\Pi_{-1}^{\mathrm{o}}, \\ \Pi_{-1}^{\mathrm{o}} & \text { if } \Pi_{1}^{\mathrm{o}}>-\Pi_{-1}^{\mathrm{o}} .\end{cases}
$$

Then you are sure to see, either to the right or to the left of the new origin, an interval followed by a longer interval. This is definitely not a property of $N^{\mathrm{o}}$ which in both directions from the origin has an exponential interval followed by an independent exponential interval. Thus $\theta_{\Pi_{\text {closest }}^{\mathrm{o}}} N^{\mathrm{o}}$ does not have the same distribution as $N^{\mathrm{o}}$, that is, shifting the origin to the closest point does not preserve the distribution of this point-stationary $N^{\mathrm{o}}$. 


\subsection{Point-maps and point-shifts}

Example 2.1 illustrates that the selection of a point to shift the origin to is the key issue when defining point-stationarity. We cannot select points in any old way. In order to discuss this problem, we need the following terminology.

Call an $\mathscr{1} \mathscr{C}^{\mathrm{o}} / \mathscr{B}^{d}$ measurable mapping $\pi$ from $M^{\mathrm{o}}$ to $\mathbb{R}^{d}$ an $N^{\mathrm{o}}$-point-map if it selects a point, that is, if

$$
\mu(\{\pi(\mu)\})=1, \quad \mu \in M^{\mathrm{o}} .
$$

Call the mapping $\theta_{\pi}$ from $M^{\mathrm{o}}$ to $M^{\mathrm{o}}$ defined by

$$
\theta_{\pi} \mu=\theta_{\pi(\mu)} \mu, \quad \mu \in M^{\mathrm{o}}
$$

an $N^{\mathrm{o}}$-point-shift.

Note that $\theta_{\pi}$ shifts the origin from a point to a point. Call $\theta_{\pi}$ the point-shift associated with $\pi$. It is readily checked that $\theta_{\pi}$ is $\mathscr{C}^{\circ} / \mathscr{L}^{\mathrm{o}}$ measurable.

When $d=1$, examples of $N^{\mathrm{o}}$-point-maps are the $\pi_{n},-\infty<n<\infty$, defined for $\mu \in M^{\mathrm{o}}$ by

$$
\pi_{n}(\mu)= \begin{cases}n \text {th } \mu \text { point to the right of } 0, & n>1 \\ 0, & n=0 \\ -n \text {th } \mu \text { point to the left of } 0, & n<-1\end{cases}
$$

The associated $N^{\circ}$-point-shifts translate the origin to the $n$th point on the right (or $-n$th on the left). Note that the random points $\Pi_{n}^{\mathrm{o}}$ introduced in Section 2.4 can be written as $\Pi_{n}^{\mathrm{o}}=\pi_{n}\left(N^{\mathrm{o}}\right)$.

When $d \geqslant 1$, an example of an $N^{\mathrm{o}}$-point-map is the shift to the closest point, $\pi_{\text {closest }}$, defined for $\mu \in M^{\mathrm{o}}$ by

$\pi_{\text {closest }}(\mu)=$ the $\mu$-point having the lexicographically highest order among the non-zero

$\mu$-points being at shortest distance from the origin.

The lexicographic rule here is just to make sure that $\pi_{\text {closest }}(\mu)$ is uniquely defined. The random point $\Pi_{\text {closest }}^{\mathrm{o}}$ in Example 2.1 can be written as $\Pi_{\text {closest }}^{\mathrm{o}}=\pi_{\text {closest }}\left(N^{\mathrm{o}}\right)$.

\subsection{What is wrong with shifting the origin to the closest point?}

In Example 2.1 shifting the origin to the closest point changed the distribution of an intervalstationary (that is, point-stationary) $N^{\mathrm{o}}$. So what is wrong with this point-shift?

In order to answer this question let us first consider another one: when $d=1$, what is so special about shifting the origin of $N^{\mathrm{o}}$ to the $n$th point on the right? The essential property of this point-shift is the following. By knowing the point-selection rule (select the $n$th point on the right) and by looking at the point pattern from the new origin you can always tell from what point you came and shift the origin back to the $n$th point on the left of the new origin: we first shift the origin of $\mu$ to $\pi_{n}(\mu)$ to obtain $\theta_{\pi_{n}} \mu$ and then the origin of $\theta_{\pi_{n}} \mu$ to 


$$
\pi_{-n}\left(\theta_{\pi_{n}} \mu\right)=-\pi_{n}(\mu)
$$

to obtain

$$
\theta_{\pi_{-n}} \theta_{\pi_{n}} \mu=\mu, \quad \mu \in M^{\mathrm{o}} .
$$

Thus $\mu$ is the only element of $M^{\mathrm{o}}$ that $\theta_{\pi_{n}}$ shifts to $\mu^{\prime}:=\theta_{\pi_{n}} \mu$. Also any element $\mu^{\prime}$ of $M^{\mathrm{o}}$ can arise from this point-shift since taking $\mu:=\theta_{\pi_{-n}} \mu^{\prime}$ yields

$$
\theta_{\pi_{n}} \mu=\mu^{\prime}
$$

Thus, shifting the origin to the $n$th point on the right is a bijective point-shift.

When $d=1$, it can be shown that an interval-stationary (that is, point-stationary) $N^{\mathrm{o}}$ is distributionally invariant under the group of all bijective $N^{\mathrm{o}}$-point-shifts (see Remark 3.1).

Shifting the origin to the closest point is wrong because this point-shift is not bijective: there can be more than one point of a point pattern having a particular point $p$ as the closest point.

\subsection{Bijective point-shifts?}

It is natural to apply bijective point-shifts when defining point-stationarity since under a bijective point-shift $\theta_{\pi}$ all points are equally important, or get equal attention, in the following sense: with $\mu \in M^{\mathrm{o}}$ fixed, the mapping

$$
p \mapsto p+\pi\left(\theta_{p} \mu\right)
$$

is a bijection from $s(\mu)$ to $s(\mu)$, that is, under this mapping each point is the image of a unique point. All this suggests that we should define point-stationarity in higher dimensions by requiring that $N^{\mathrm{o}}$ is distributionally invariant under bijective $N^{\mathrm{o}}$-point-shifts.

But for this definition to make sense we must find some bijective point-shifts, other than the trivial $\theta_{0}$. Having searched for such point-shifts for quite some time, the author doubts that they exist and therefore makes the following guess, without further motivation.

Conjecture 2.1. No nontrivial bijective $N^{\mathrm{o}}$-point-shifts exist when $d>1$.

If this is true, how then can we define point-stationarity in higher dimensions? The answer is that bijective point-shifts exist if we consider $N^{\mathrm{o}}$ against an independent stationary background. We explain what this means in the next section.

\section{Definition of point-stationarity}

In order to highlight the point-stationarity problem, we have up to now avoided saying that the point process $N^{\mathrm{o}}$ will be regarded in association with a random field $X^{\mathrm{o}}$. Considering $N^{\mathrm{o}}$ jointly with $X^{\mathrm{o}}$ does not solve our problem but is conceptually a step in the right direction. 


\subsection{The associated random fields $X$ and $X^{\circ}$}

We shall henceforth consider a pair $(N, X)$ where $N$ is a simple counting process and

$$
X=\left(X_{s}\right)_{s \in \mathbb{R}^{d}}
$$

is a random field with an arbitrary state space $(E, \mathscr{E})$ and path space $(H, \mathscr{H})$, where $H$ is a shift-invariant subset of $E^{\mathbb{R}^{d}}$ and $\mathscr{H}$ is the $\sigma$-algebra on $H$ generated by the projection mappings taking $x=\left(x_{s}\right)_{s \in \mathbb{R}^{d}}$ in $H$ to $x_{t}$ in $E, t \in \mathbb{R}^{d}$.

In order to be able to apply random shifts we need the minimal regularity condition (satisfied in the standard settings, for instance when $(E, \mathscr{E})$ is Polish and the paths rightcontinuous) that $X$ is canonically measurable, that is, the mapping from $H \times \mathbb{R}^{d}$ to $E$ taking $(x, t)$ to $x_{t}$ is $\mathscr{H} \otimes \mathscr{B}^{d} / \mathscr{E}$ measurable. For $t \in \mathbb{R}^{d}$, let $\theta_{t}$ denote the shift or site-shift from $H$ to $H$ defined by

$$
\theta_{t} x=\left(x_{t+s}\right)_{s \in \mathbb{R}^{d}}, \quad x \in H .
$$

Canonical measurability is equivalent to the mapping from $H \times \mathbb{R}^{d}$ to $H$ taking $(x, t)$ to $\theta_{t} x$ being $\mathscr{H} \otimes \mathscr{B}^{d} / \mathscr{H}$ measurable (shift-measurability).

(Considering $N$ in association with a random field is equivalent to considering a marked point process in the following sense. When $X$ is given we could define the mark of a point $\Pi$ to be $\theta_{\Pi} X$. Conversely, when the marks are given we could define $X$ by letting $X_{s}$ be the marked point process with origin shifted to $s$.)

We shall not assume any functional connection between $N$ and $X$. At one extreme, $N$ could be determined by $X$. For instance, when $d=1$, the points could be the times when a stochastic process enters a given state. At the other extreme, $X$ could be identically constant, which boils down to regarding $N$ alone (not in association with any random field) as we did in Section 2 .

When we consider a random field in association with $N^{\mathrm{o}}$ we shall denote it $X^{\mathrm{o}}$. Thus the ${ }^{\mathrm{o}}$ on $X^{\mathrm{o}}$ is not to indicate a property of $X^{\mathrm{o}}$. It only indicates that $X^{\mathrm{o}}$ is considered jointly with $N^{\mathrm{o}}$ (while as before the ${ }^{\mathrm{o}}$ on $N^{\mathrm{o}}$ is to indicate that $N^{\mathrm{o}}$ has a point at the origin).

\subsection{Extended point-maps and point-shifts}

Let $\theta_{t}$ denote the shift or site-shift from $M \times H$ to $M \times H$ defined, for $t \in \mathbb{R}^{d}$, by

$$
\theta_{t}(\mu, x)=\left(\theta_{t} \mu, \theta_{t} x\right), \quad(\mu, x) \in M \times H .
$$

Call an $\mathscr{L}^{\mathrm{o}} \otimes \mathscr{H} / \mathscr{B}^{d}$ measurable mapping $\pi$ from $M^{\mathrm{o}} \times H$ to $\mathbb{R}^{d}$ an $\left(N^{\mathrm{o}}, X^{\mathrm{o}}\right)$-point-map if it selects a point, that is, if

$$
\mu(\{\pi(\mu, x)\})=1, \quad(\mu, x) \in M^{\mathrm{o}} \times H .
$$

Call the mapping $\theta_{\pi}$ from $M^{\mathrm{o}} \times H$ to $M^{\mathrm{o}} \times H$ defined by

$$
\theta_{\pi}(\mu, x)=\theta_{\pi(\mu, x)}(\mu, x), \quad(\mu, x) \in M^{\mathrm{o}} \times H,
$$

an $\left(N^{\mathrm{o}}, X^{\mathrm{o}}\right)$-point-shift.

Note that $\theta_{\pi}$ shifts the origin from a point to a point. Call $\theta_{\pi}$ the point-shift associated 
with $\pi$. The $\mathscr{C}^{\circ} \otimes \mathscr{H} / \mathscr{C}^{\circ} \otimes \mathscr{H}$ measurability of $\theta_{\pi}$ follows from the canonical measurability of $X^{\mathrm{o}}$.

In order to distinguish $\left(N^{\mathrm{o}}, X^{\mathrm{o}}\right)$-point-maps and -shifts from $N^{\mathrm{o}}$-point-maps and -shifts we shall sometimes call them extended point-maps and extended point-shifts.

\subsection{The point-stationarity problem for $\left(N^{\mathrm{o}}, X^{\mathrm{o}}\right)$}

The pair $(N, X)$ is stationary if it looks the same from all observation sites, that is,

$$
\theta_{t}(N, X) \stackrel{D}{=}(N, X), \quad t \in \mathbb{R}^{d} .
$$

Similarly, (as in the case of $N^{\mathrm{o}}$ alone) it would be natural to say that the pair $\left(N^{\mathrm{o}}, X^{\mathrm{o}}\right)$ is point-stationary if it looks the same from all observation points.

When $d=1$, point-stationarity means that $\left(N^{\mathrm{o}}, X^{\mathrm{o}}\right)$ is cycle-stationary, that is, the points of $N^{\mathrm{o}}$ split $X^{\mathrm{o}}$ into a stationary sequence of cycles. This definition of point-stationarity, when $d=1$, can be rewritten as

$$
\theta_{\Pi_{n}^{\mathrm{o}}}\left(N^{\mathrm{o}}, X^{\mathrm{o}}\right) \stackrel{D}{=}\left(N^{\mathrm{o}}, X^{\mathrm{o}}\right), \quad n \in \mathbb{Z},
$$

and it can be shown that this is equivalent to $\left(N^{\mathrm{o}}, X^{\mathrm{o}}\right)$ being distributionally invariant under the group of all bijective $\left(N^{\mathrm{o}}, X^{\mathrm{o}}\right)$-point-shifts (again see Remark 3.1).

Under a bijective $\left(N^{\mathrm{o}}, X^{\mathrm{o}}\right)$-point-shift $\theta_{\pi}$ all points are equally important, or get equal attention, in the same sense as in the $N^{\mathrm{o}}$ case: with $(\mu, x) \in M^{\mathrm{o}} \times H$ fixed, the mapping

$$
p \mapsto p+\pi\left(\theta_{p}(\mu, x)\right)
$$

is a bijection from $s(\mu)$ to $s(\mu)$, that is, under this mapping each point is the image of a unique point.

This again suggests that we should define point-stationarity for $d>1$ by requiring that $\left(N^{\mathrm{o}}, X^{\mathrm{o}}\right)$ is distributionally invariant under bijective $\left(N^{\mathrm{o}}, X^{\mathrm{o}}\right)$-point-shifts. Even if Conjecture 2.1 is true, non-trivial bijective $\left(N^{\mathrm{o}}, X^{\mathrm{o}}\right)$-point-shifts might exist for certain random fields $X^{\mathrm{o}}$. Indeed, we shall give an example in Section 3.6.

Although this will not give us a definition of point-stationarity for arbitrary pairs $\left(N^{\mathrm{o}}, X^{\mathrm{o}}\right)$ it is a step half-way towards the solution.

\subsection{Intuitive motivation of the solution}

The solution to the point-stationarity problem is to consider $\left(N^{\mathrm{o}}, X^{\mathrm{o}}\right)$ against any independent stationary background, that is, consider $\left(N^{\mathrm{o}}, X^{\mathrm{o}}\right)$ jointly with an arbitrary independent stationary (canonically measurable) random field

$$
Y^{\mathrm{o}}=\left(Y_{s}^{\mathrm{o}}\right)_{s \in \mathbb{R}^{d}} .
$$

Let $(L, \mathscr{B})$ be the path space of $Y^{\circ}$. Note that the ${ }^{\circ}$ on $Y^{\circ}$ (like the ${ }^{\circ}$ on $X^{\circ}$ ) is only to indicate that $Y^{\mathrm{o}}$ is considered in association with $N^{\mathrm{o}}$ and does not imply that $Y^{\mathrm{o}}$ and $N^{\mathrm{o}}$ are 
functionally connected (while the ${ }^{\mathrm{o}}$ on the point process $N^{\mathrm{o}}$ itself indicates that $N^{\mathrm{o}}$ has a point at the origin).

Intuitively, if the triple $\left(N^{\mathrm{o}}, X^{\mathrm{o}}, Y^{\mathrm{o}}\right)$ looks the same from all the points of $N^{\mathrm{o}}$ then so, in particular, does the pair $\left(N^{\mathrm{o}}, X^{\mathrm{o}}\right)$. Conversely, if $\left(N^{\mathrm{o}}, X^{\mathrm{o}}\right)$ looks the same from all the points of $N^{\mathrm{o}}$ then so will $\left(N^{\mathrm{o}}, X^{\mathrm{o}}, Y^{\mathrm{o}}\right)$ because (due to the stationarity of $\left.Y^{\mathrm{o}}\right) Y^{\mathrm{o}}$ looks the same from all random sites that are independent of $Y^{\circ}$ and thus (due to the independence of $Y^{\mathrm{o}}$ and $\left(N^{\mathrm{o}}, X^{\mathrm{o}}\right)$ and the fact that $\left(N^{\mathrm{o}}, X^{\mathrm{o}}\right)$ looks the same from all the points of $\left.N^{\mathrm{o}}\right)$ the triple $\left(N^{\mathrm{o}}, X^{\mathrm{o}}, Y^{\mathrm{o}}\right)$ should look the same from all points of $N^{\mathrm{o}}$.

In other words, $\left(N^{\mathrm{o}}, X^{\mathrm{o}}\right)$ should be point-stationary if and only if $\left(N^{\mathrm{o}}, X^{\mathrm{o}}, Y^{\mathrm{o}}\right)$ is pointstationary.

\subsection{Solution of the point-stationarity problem}

This suggests that we call the pair $\left(N^{\mathrm{o}}, X^{\mathrm{o}}\right)$ point-stationary if the triple $\left(N^{\mathrm{o}}, X^{\mathrm{o}}, Y^{\mathrm{o}}\right)$ is distributionally invariant under all bijective $\left(N^{\mathrm{o}}, X^{\mathrm{o}}, Y^{\mathrm{o}}\right)$-point-shifts for all canonically measurable random fields $Y^{\mathrm{o}}$ which are stationary and independent of $\left(N^{\mathrm{o}}, X^{\mathrm{o}}\right)$. The above discussion motivates this definition intuitively, while the theory established in the upcoming sections motivates it practically. Here is the definition stated in full detail.

Definition 3.1. Call $\left(N^{\mathrm{o}}, X^{\mathrm{o}}\right)$ point-stationary if

$$
\theta_{\Pi^{\mathrm{o}}}\left(N^{\mathrm{o}}, X^{\mathrm{o}}, Y^{\mathrm{o}}\right) \stackrel{D}{=}\left(N^{\mathrm{o}}, X^{\mathrm{o}}, Y^{\mathrm{o}}\right)
$$

for all random points $\Pi^{\mathrm{o}}$ of the form

$$
\Pi^{\mathrm{o}}=\pi\left(N^{\mathrm{o}}, X^{\mathrm{o}}, Y^{\mathrm{o}}\right),
$$

where $Y^{\mathrm{o}}$ is any canonically measurable random field which is stationary and independent of $\left(N^{\mathrm{o}}, X^{\mathrm{o}}\right)$ and $\pi$ is any $\left(N^{\mathrm{o}}, X^{\mathrm{o}}, Y^{\mathrm{o}}\right)$-point-map (that is, $\pi$ is an $\mathscr{C}^{\mathrm{o}} \otimes \mathscr{H} \otimes \mathscr{B} / \mathscr{B}^{d}$ measurable mapping from $M^{\mathrm{o}} \times H \times L$ to $\mathbb{R}^{d}$ which selects a point:

$$
\left.\mu(\{\pi(\mu, x, y)\})=1, \quad(\mu, x, y) \in M^{\mathrm{o}} \times H \times L\right)
$$

such that the associated point-shift (that is, the $\mathscr{L}^{\circ} \otimes \mathscr{H} \otimes \mathscr{B} / \mathscr{L}^{\circ} \otimes \mathscr{H} \otimes \mathscr{B}$ measurable mapping $\theta_{\pi}$ from $M^{0} \times H \times L$ to $M^{0} \times H \times L$ defined by

$$
\left.\theta_{\pi}(\mu, x, y)=\theta_{\pi(\mu, x, y)}(\mu, x, y), \quad(\mu, x, y) \in M^{\mathrm{o}} \times H \times L\right)
$$

is a bijection.

Remark 3.1. When $d=1$, this point-stationarity definition is equivalent to the apparently weaker property of cycle-stationarity since both properties are equivalent to (4.3) in Theorem 4.1 below.

Remark 3.2. Definition 3.1 is equivalent (see Lemma 4.1) to the apparently weaker condition 


$$
\theta_{\Pi^{\mathrm{o}}}\left(N^{\mathrm{o}}, X^{\mathrm{o}}\right) \stackrel{D}{=}\left(N^{\mathrm{o}}, X^{\mathrm{o}}\right)
$$

for all random points

$$
\Pi^{\mathrm{o}}=\kappa_{n}\left(N^{\mathrm{o}}, Y^{\mathrm{o}}\right),
$$

where $Y^{\mathrm{o}}$ and $\kappa_{n}, n \in \mathbb{Z}$, are from the family of random fields and point-shifts (indexed by $h>0$ ) defined in the next subsection.

\subsection{A key example of extended bijective point-shifts}

We shall now construct a random field $Y^{\mathrm{o}}$ and a two-sided sequence of $\left(N^{\mathrm{o}}, Y^{\mathrm{o}}\right)$-point-maps $\kappa_{n}, n \in \mathbb{Z}$, such that the associated $\left(N^{\mathrm{o}}, Y^{\mathrm{o}}\right)$-point-shifts are bijections. Both $Y^{\mathrm{o}}$ and the point-maps will depend on a fixed constant $h>0$. Thus we are really defining a family of random fields and point-shifts indexed by $h>0$, although we suppress the parameter $h$ in the notation.

We start by constructing $Y^{\mathrm{o}}$ which will simply represent the stationary point-pattern $h \mathbb{Z}^{d}-h U$, where $U$ is uniformly distributed on $\left[-\frac{1}{2}, \frac{1}{2}\right)^{d}$. Let $b=\left(b_{s}\right)_{s \in \mathbb{R}^{d}}$ be the fixed function from $\mathbb{R}^{d}$ to $\mathbb{R}^{d}$ defined by

$$
b_{s}=\text { the vector from the site } s \text { to the closest element of } h \mathbb{Z}^{d} \text {. }
$$

If there are more than one such $h \mathbb{Z}^{d}$-elements, let $b_{s}$ be the vector to the element of highest lexicographic order (thus $b_{s}$ is right-continuous in all the $d$ coordinates of $s$ ).

Let $Y^{\mathrm{o}}$ have state space $\left(\mathbb{R}^{d}, \mathscr{B}^{d}\right)$ and have paths in

$$
L=\left\{\theta_{t} b: t \in[-h / 2, h / 2)^{d}\right\}=\left\{\theta_{t} b: t \in \mathbb{R}^{d}\right\} .
$$

Let $U$ be a random site which is uniformly distributed on $\left[-\frac{1}{2}, \frac{1}{2}\right)^{d}$ and independent of $\left(N^{\mathrm{o}}, X^{\mathrm{o}}\right)$, and define

$$
Y^{\mathrm{o}}=\theta_{U h} b .
$$

Clearly $Y^{\mathrm{o}}$ is stationary and independent of $\left(N^{\mathrm{o}}, X^{\mathrm{o}}\right)$, and canonical measurability follows from right-continuity of the paths.

We now turn to constructing the bijective $\left(N^{\mathrm{o}}, Y^{\mathrm{o}}\right)$-point-maps $\kappa_{n}, n \in \mathbb{Z}$. Fix $(\mu, y) \in M^{0} \times L$. Call a site $t \in \mathbb{R}^{d}$ such that $y_{t}=0$ a $y$-centre and the associated set $t+[-h / 2, h / 2)^{d}$ a $y$-box. Note that $y_{0}$ is the centre of the $y$-box containing the origin. Let $k$ be the number of $\mu$-points in that box,

$$
k=\mu\left(y_{0}+[-h / 2, h / 2)^{d}\right),
$$

$\left(k \geqslant 1\right.$ since $0 \in s(\mu)$ and $\left.y_{0} \in(-h / 2, h / 2]^{d}\right)$. Let $p_{0}, \ldots, p_{k-1}$ be the $\mu$-points in $s(\mu) \cap\left(y_{0}+[-h / 2, h / 2)^{d}\right)$ ordered lexicographically. Let $m$ denote the index of the $\mu$-point at the origin, that is, $p_{m}=0$. For $n \in \mathbb{Z}$, put

$$
\kappa_{n}(\mu, y)=p_{m+n}(\bmod k),
$$

where 


$$
(m+n) \bmod k=\inf (m+n-k \mathbb{Z}) \cap[0, \infty) .
$$

That is, $\kappa_{n}(\mu, y)$ is the $n$th point after the point at the origin (if $n<0$, interpret this as the $-n$th point before the point at the origin) in a circular enumeration of the points in the box containing the origin. Thus the point at the origin is the $n$th point before $\kappa_{n}(\mu, y)$ in that same circular enumeration. Thus

$$
\theta_{k_{-n}} \theta_{k_{n}}(\mu, y)=(\mu, y) \quad(\mu, y) \in M^{\mathrm{o}} \times L .
$$

Thus $(\mu, y)$ is the only element of $M^{\mathrm{o}} \times L$ that $\theta_{\kappa_{n}}$ shifts to $\left(\mu^{\prime}, y^{\prime}\right):=\theta_{\kappa_{n}}(\mu, y)$. Also any element $\left(\mu^{\prime}, y^{\prime}\right)$ of $M^{\mathrm{o}} \times L$ can arise from $\theta_{\kappa_{n}}$ since taking $(\mu, y):=\theta_{\kappa_{-n}}\left(\mu^{\prime}, y^{\prime}\right)$ yields $\theta_{\kappa_{n}}(\mu, y)=\left(\mu^{\prime}, y^{\prime}\right)$. Thus $\theta_{\kappa_{n}}$ is bijective.

\section{Palm characterization of point-stationarity}

In this section we establish a pleasant characterization of point-stationarity in terms of nonrandom site-shifts. We shall call it a Palm characterization because it is the key to the Palm dualities presented in Sections 8 and 9. At the end of the section we state a dual Palm characterization of stationarity.

We start by introducing an important concept, so-called Voronoi cells, which play in some respects the same role in higher dimensions as the intervals between points in one dimension.

\subsection{Voronoi cells}

Consider a point pattern in $\mathbb{R}^{d}$ represented by a simple counting measure $\mu \in M$. Note that the point pattern $s(\mu)$ need not have a point at the origin. With each point $p \in s(\mu)$ associate the set of sites $t \in \mathbb{R}^{d}$ which are strictly closer to $p$ than to any other point. This set is the open Voronoi cell with point $p$. Define the Voronoi cells themselves by extending the open Voronoi cells in such a way that a site $t \in \mathbb{R}^{d}$ which is at equal minimal distance to two or more points belongs to the cell with point $p$ having the highest lexicographic order.

Thus with each point $p \in s(\mu)$ there is associated a Voronoi cell. There are finitely or countably many of these Voronoi cells, they are disjoint, and their union is $\mathbb{R}^{d}$. In other words, the Voronoi cells form a finite or countable partition of $\mathbb{R}^{d}$.

Note that, when $d=1$, the Voronoi cells are intervals with the points in the interior while one usually considers intervals with the points at the ends. For further information on Voronoi cells, see for instance Okabe et al. (1992).

In what follows the Voronoi cell containing the origin is of key importance. For $N$ put

$$
C_{0}=\text { the Voronoi cell of } N \text { which contains the origin }
$$

and

$$
\Pi_{0}=\text { the } N \text {-point of } C_{0} \text {. }
$$

For $N^{\mathrm{o}}$ put 
$C_{0}^{\mathrm{o}}=$ the Voronoi cell of $N^{\mathrm{o}}$ which contains the point at the origin.

\subsection{Shifting the origin to and from $\Pi_{0}$}

From now on the pairs $(N, X)$ and $\left(N^{\mathrm{o}}, X^{\mathrm{o}}\right)$ will be functionally linked as follows. Regard $(N, X)$ as given. Define $\left(N^{\mathrm{o}}, X^{\mathrm{o}}\right)$ and a $C_{0}^{\mathrm{o}}$-valued random site $S$ by

$$
\left(N^{\mathrm{o}}, X^{\mathrm{o}}\right) \equiv \theta_{\Pi_{0}}(N, X), \quad \text { and } S \equiv-\Pi_{0} .
$$

Thus $\left(\left(N^{\mathrm{o}}, X^{\mathrm{o}}\right), S\right)$ is $((N, X), 0)$ seen from $\Pi_{0}$.

Conversely, regard $\left(N^{\mathrm{o}}, X^{\mathrm{o}}\right)$ and a $C_{0}^{\mathrm{o}}$-valued random site $S$ as given. Define $(N, X)$ by

$$
(N, X) \equiv \theta_{S}\left(N^{\mathrm{o}}, X^{\mathrm{o}}\right)
$$

(this implies that $\left.\Pi_{0} \equiv-S\right)$. Thus $(N, X)$ is $\left(N^{\mathrm{o}}, X^{\mathrm{o}}\right)$ seen from $S$.

Under this functional one-to-one correspondence between $(N, X)$ and $\left(\left(N^{\mathrm{o}}, X^{\mathrm{o}}\right), S\right)$ we have

$$
C_{0}^{\mathrm{o}} \equiv C_{0}-\Pi_{0}
$$

or, equivalently,

$$
C_{0} \equiv C_{0}^{\mathrm{o}}-S
$$

Thus, in particular, the Voronoi cells $C_{0}$ and $C_{0}^{\mathrm{o}}$ have the same volume:

$$
\lambda\left(C_{0}^{\mathrm{o}}\right) \equiv \lambda\left(C_{0}\right)
$$

where $\lambda$ is Lebesgue measure on $\left(\mathbb{R}^{d}, \mathscr{B}^{d}\right)$.

\subsection{The Palm characterization of point-stationarity}

We shall now show that point-stationarity of the pair $\left(N^{\mathrm{o}}, X^{\mathrm{o}}\right)$ means that if we shift the origin to a site selected uniformly at random in $C_{0}^{\mathrm{o}}$ then the pair will look the same from all observation sites provided we volume-bias by the volume of $C_{0}^{\mathrm{o}}$.

Theorem 4.1. Let $\left(N^{\mathrm{o}}, X^{\mathrm{o}}\right), S$ and $(N, X)$ be linked as in Section 4.2, and suppose $\lambda\left(C_{0}\right)$ is finite with probability one. Then

$$
\left(N^{\mathrm{o}}, X^{\mathrm{o}}\right) \text { is point-stationary }
$$

and

$$
\text { the conditional distribution of } S \text { given }\left(N^{\mathrm{o}}, X^{\mathrm{o}}\right) \text { is uniform on } C_{0}^{\mathrm{o}}
$$

if and only if

$$
\mathrm{E}\left[f\left(\theta_{t}(N, X)\right) \lambda\left(C_{0}\right)\right]=\mathrm{E}\left[f(N, X) \lambda\left(C_{0}\right)\right], \quad f \in \mathscr{N} \otimes \otimes \mathscr{H}^{+}, t \in \mathbb{R}^{d} .
$$

We prove Theorem 4.1 in the next three subsections. 


\subsection{First part of proof of Theorem 4.1: (4.1) and (4.2) imply (4.3)}

We shall first assume that (4.1) and (4.2) hold and show that this implies (4.3). Take $t \in \mathbb{R}^{d}$ and $f \in \mathscr{C} \otimes \mathscr{H}^{+}$and use the conditional uniformity (4.2), and $\lambda\left(C_{0}^{\mathrm{o}}\right) \equiv \lambda\left(C_{0}\right)$, to obtain the second identity in

$$
\begin{aligned}
\mathrm{E}\left[\lambda\left(C_{0}\right) f\left(\theta_{t}(N, X)\right)\right] & =\mathrm{E}\left[\lambda\left(C_{0}\right) f\left(\theta_{t+S}\left(N^{\mathrm{o}}, X^{\mathrm{o}}\right)\right)\right] \\
& =\mathrm{E}\left[\int_{t+C_{0}^{\mathrm{o}}} f\left(\theta_{s}\left(N^{\mathrm{o}}, X^{\mathrm{o}}\right)\right) \mathrm{d} s\right] .
\end{aligned}
$$

Let $\kappa_{n}$ and $Y^{\mathrm{o}}$ be as in Section 3.6; furthermore, let $C^{n}$ denote the Voronoi cell of $N^{\mathrm{o}}$ associated with the point $\kappa_{n}\left(N^{\mathrm{o}}, Y^{\mathrm{o}}\right)$,

$$
K=N^{\mathrm{o}}\left(Y_{0}^{\mathrm{o}}+[-h / 2, h / 2)^{d}\right)
$$

denote the number of $N^{\mathrm{o}}$-points in the $Y^{\mathrm{o}}$-box containing the origin, and

$$
\mathrm{A}=\bigcup_{\mathrm{n}=\mathrm{o}}^{K-1} \mathrm{C}^{\mathrm{n}}
$$

denote the union of cells with points in the $Y^{\mathrm{o}}$-box containing the origin.

Note that the number of $N^{\mathrm{o}}$-points in the box containing the origin remains (since the point-map $\kappa_{n}$ selects a point in that box) the same after the shift by $\theta_{\kappa_{n}}$, namely $K$. Thus $1_{\{K=k\}} \int\left(t+C_{0}^{\mathrm{o}}\right) \cap C^{n} f\left(\theta_{s}\left(N^{\mathrm{o}}, X^{\mathrm{o}}\right)\right) \mathrm{d} s$ is obtained from $\left(N^{\mathrm{o}}, X^{\mathrm{o}}, Y^{\mathrm{o}}\right)$ by the same function in $\mathscr{L}^{\mathrm{o}} \otimes \mathscr{H} \mathbb{B} \otimes \mathscr{D}^{+}$as $1_{\{K=k\}} \int\left(t+C^{-n}\right) \cap C_{0}^{\mathrm{o}} f\left(\theta_{s}\left(N^{\mathrm{o}}, X^{\mathrm{o}}\right)\right) \mathrm{d} s$ from $\theta_{\kappa_{n}}\left(N^{\mathrm{o}}, X^{\mathrm{o}}, Y^{\mathrm{o}}\right)$. Due to (4.1), $\left(N^{\mathrm{o}}, X^{\mathrm{o}}, Y^{\mathrm{o}}\right)$ and $\theta_{\kappa_{-n}}\left(N^{\mathrm{o}}, X^{\mathrm{o}}, Y^{\mathrm{o}}\right)$ have the same distribution, and thus

$$
\mathrm{E}\left[1_{\{K=k\}} \int_{\left(t+C_{0}^{\mathrm{o}}\right) \cap C^{n}} f\left(\theta_{s}\left(N^{\mathrm{o}}, X^{\mathrm{o}}\right)\right) \mathrm{d} s\right]=\mathrm{E}\left[1_{\{K=k\}} \int_{\left(t+C^{-n}\right) \cap C_{0}^{\mathrm{o}}} f\left(\theta_{s}\left(N^{\mathrm{o}}, X^{\mathrm{o}}\right)\right) \mathrm{d} s\right] .
$$

Recall that $A$ is the union of $C^{n}$ over $0 \leqslant n<K$ and note that $A$ is also the union of $C^{-n}$ over $0 \leqslant n<K$. Thus summing first over $0 \leqslant n<k$ and then over $1 \leqslant k<\infty$ yields

$$
\mathrm{E}\left[\int_{\left(t+C_{0}^{\mathrm{o}}\right) \cap A} f\left(\theta_{s}\left(N^{\mathrm{o}}, X^{\mathrm{o}}\right)\right) \mathrm{d} s\right]=\mathrm{E}\left[\int_{(t+A) \cap C_{0}^{\mathrm{o}}} f\left(\theta_{s}\left(N^{\mathrm{o}}, X^{\mathrm{o}}\right)\right) \mathrm{d} s\right] .
$$

Now $A$ in fact depends on the parameter $h$ and expands to the union of all the Voronoi cells as $h \rightarrow \infty$. Thus both $A$ and $t+A$ expand to $\mathbb{R}^{d}$ as $h \rightarrow \infty$. Thus by monotone convergence

$$
\mathrm{E}\left[\int_{t+C_{0}^{\mathrm{o}}} f\left(\theta_{s}\left(N^{\mathrm{o}}, X^{\mathrm{o}}\right)\right) \mathrm{d} s\right]=\mathrm{E}\left[\int_{C_{0}^{\mathrm{o}}} f\left(\theta_{s}\left(N^{\mathrm{o}}, X^{\mathrm{o}}\right)\right) \mathrm{d} s\right] .
$$

Apply this to (4.4) to obtain that $\mathrm{E}\left[\lambda\left(C_{0}\right) f\left(\theta_{t}(N, X)\right)\right]$ does not depend on $t$, that is, (4.3) holds. 


\subsection{Middle part of proof of Theorem 4.1: (4.3) implies (4.2)}

We shall now assume that (4.3) holds and show that this implies (4.2). For $t \in \mathbb{R}^{d}$, write $C_{t}$ for the Voronoi cell of $N$ containing the site $t$, and $\Pi_{t}$ for the $N$-point of $C_{t}$. From (4.3) and $P\left(\lambda\left(C_{0}\right)<\infty\right)=1$ we have

$$
\mathrm{E}\left[1_{\left\{\lambda\left(C_{t}\right)=\infty\right\}} \lambda\left(C_{0}\right)\right]=\mathrm{E}\left[1_{\left\{\lambda\left(C_{0}\right)=\infty\right\}} \lambda\left(C_{0}\right)\right]=0,
$$

which, together with $\lambda\left(C_{0}\right)>0$, yields

$$
P\left(\lambda\left(C_{t}\right)<\infty\right)=1, \quad t \in \mathbb{R}^{d} .
$$

Let $g$ be a non-negative measurable real-valued function defined on $\mathbb{R}^{d}$ and let $f \in$ $\mathscr{C} \otimes \otimes \mathscr{H}^{+}$. Note that $\lambda\left(C_{0}\right)^{-1} g(S) f\left(N^{\mathrm{o}}, X^{\mathrm{o}}\right)$ is obtained from $(N, X)$ by the same function in $\mathscr{C} \otimes \mathscr{H}^{+}$as $\lambda\left(C_{t}\right)^{-1} g\left(t-\Pi_{t}\right) f\left(\theta_{\Pi_{t}}(N, X)\right)$ from $\theta_{t}(N, X)$. Due to (4.3), this implies that

$$
\mathrm{E}\left[g(S) f\left(N^{\mathrm{o}}, X^{\mathrm{o}}\right)\right]=\mathrm{E}\left[\lambda\left(C_{0}\right) \lambda\left(C_{t}\right)^{-1} g\left(t-\Pi_{t}\right) f\left(\theta_{\Pi_{t}}(N, X)\right)\right] .
$$

Integrating over $0 \leqslant t<1$ and interchanging integration and expectation yields

$$
\mathrm{E}\left[g(S) f\left(N^{\mathrm{o}}, X^{\mathrm{o}}\right)\right]=\mathrm{E}\left[\lambda\left(C_{0}\right) \int_{0 \leqslant t<1} \lambda\left(C_{t}\right)^{-1} g\left(t-\Pi_{t}\right) f\left(\theta_{\Pi_{t}}(N, X)\right) \mathrm{d} t\right] .
$$

Take $i \in \mathbb{Z}^{d}$ and note that

$$
\int_{0 \leqslant t<1} 1_{\left\{i \leqslant \Pi_{t}<i+1\right\}} \lambda\left(C_{t}\right)^{-1} g\left(t-\Pi_{t}\right) f\left(\theta_{\Pi_{t}}(N, X)\right) \mathrm{d} t
$$

is obtained from $(N, X)$ by the same function in $\mathscr{C} \otimes \mathscr{H}^{+}$as

$$
\int_{-i \leqslant t<-i+1} 1_{\left\{0 \leqslant \Pi_{t}<1\right\}} \lambda\left(C_{t}\right)^{-1} g\left(t-\Pi_{t}\right) f\left(\theta_{\Pi_{t}}(N, X)\right) \mathrm{d} t
$$

from $\theta_{-i}(N, X)$. Applying (4.3), with $t$ replaced by $-i$, yields

$$
\begin{aligned}
& \mathrm{E}\left[\lambda\left(C_{0}\right) \int_{0 \leqslant t<1} 1_{\left\{i \leqslant \Pi_{t}<i+1\right\}} \lambda\left(C_{t}\right)^{-1} g\left(t-\Pi_{t}\right) f\left(\theta_{\Pi_{t}}(N, X)\right) \mathrm{d} t\right] \\
& \quad=\mathrm{E}\left[\lambda\left(C_{0}\right) \int_{-i \leqslant t<-i+1} 1_{\left\{0 \leqslant \Pi_{t}<1\right\}} \lambda\left(C_{t}\right)^{-1} g\left(t-\Pi_{t}\right) f\left(\theta_{\Pi_{t}}(N, X)\right) \mathrm{d} t\right] .
\end{aligned}
$$

Sum over $i \in \mathbb{Z}^{d}$ to obtain, due to (4.5) and since $\mathbb{R}^{d}$ is the union of the disjoint sets, [- $i$, $-i+1)^{d}$

$$
\mathrm{E}\left[g(S) f\left(N^{\mathrm{o}}, X^{\mathrm{o}}\right)\right]=\mathrm{E}\left[\lambda\left(C_{0}\right) \int_{t \in \mathbb{R}^{d}} 1_{\left\{0 \leqslant \Pi_{t}<1\right\}} \lambda\left(C_{t}\right)^{-1} g\left(t-\Pi_{t}\right) f\left(\theta_{\Pi_{t}}(N, X)\right) \mathrm{d} t\right] .
$$

Since $\left\{t \in \mathbb{R}^{d}: 0 \leqslant \Pi_{t}<1\right\}$ is the disjoint union of $C_{p}$ over the points $p$ in $[0,1)^{d}$ and since

$$
\begin{aligned}
\lambda\left(C_{t}\right) & =\lambda\left(C_{p}\right) \quad \text { and } \quad f\left(\theta_{\Pi_{t}}(N, X)\right)=f\left(\theta_{p}(N, X)\right), \quad t \in C_{p}, \\
g\left(t-\Pi_{t}\right) & =g(t-p), \quad t \in C_{p},
\end{aligned}
$$


we obtain

$$
\mathrm{E}\left[g(S) f\left(N^{\mathrm{o}}, X^{\mathrm{o}}\right)\right]=\mathrm{E}\left[\lambda\left(C_{0}\right) \sum_{p \in s(N) \cap[0,1)^{d}} f\left(\theta_{p}(N, X)\right) \lambda\left(C_{p}\right)^{-1} \int_{t \in C_{p}} g(t-p) \mathrm{d} t\right] .
$$

Taking $g \equiv 1$ yields

$$
\mathrm{E}\left[f\left(N^{\mathrm{o}}, X^{\mathrm{o}}\right)\right]=\mathrm{E}\left[\lambda\left(C_{0}\right) \sum_{p \in s(N) \cap[0,1)^{d}} f\left(\theta_{p}(N, X)\right)\right], \quad f \in \mathscr{l} b \otimes \mathscr{H}^{+} .
$$

Replacing $f\left(N^{\mathrm{o}}, X^{\mathrm{o}}\right)$ by $f\left(N^{\mathrm{o}}, X^{\mathrm{o}}\right) \lambda\left(C_{0}\right)^{-1} \int_{t \in C_{0}^{\mathrm{o}}} g(t) \mathrm{d} t$ in (4.7) yields

$$
\begin{aligned}
& \mathrm{E}\left[f\left(N^{\mathrm{o}}, X^{\mathrm{o}}\right) \lambda\left(C_{0}\right)^{-1} \int_{t \in C_{0}^{\mathrm{o}}} g(t) \mathrm{d} t\right] \\
& \quad=\mathrm{E}\left[\lambda\left(C_{0}\right) \sum_{p \in s(N) \cap[0,1)^{d}} f\left(\theta_{p}(N, X)\right) \lambda\left(C_{p}\right)^{-1} \int_{t \in C_{p}} g(t-p) \mathrm{d} t\right] .
\end{aligned}
$$

Compare this and (4.6) to obtain

$$
\mathrm{E}\left[g(S) f\left(N^{\mathrm{o}}, X^{\mathrm{o}}\right)\right]=\mathrm{E}\left[f\left(N^{\mathrm{o}}, X^{\mathrm{o}}\right) \lambda\left(C_{0}\right)^{-1} \int_{t \in C_{0}^{\mathrm{o}}} g(t) \mathrm{d} t\right], \quad g \in \mathscr{B}^{d^{+}}, f \in \mathscr{C} \boldsymbol{\mathscr { C }} \otimes \mathscr{H}^{+},
$$

which is equivalent to (4.2).

\subsection{Final part of proof of Theorem 4.1: (4.3) implies (4.1)}

Finally, we assume that (4.3) holds and show that this implies (4.1). Let $Y^{\circ}$ and $\pi$ be as in Definition 3.1. Assume also that $Y^{\circ}$ is independent of $(N, X)$, that is, not only independent of $\left(N^{\mathrm{o}}, X^{\mathrm{o}}\right)$ but also of $\left(\left(N^{\mathrm{o}}, X^{\mathrm{o}}\right), S\right)$. (This is no restriction since if this were not the case we could replace $Y^{\circ}$ by a random field which is independent of $(N, X)$ and has the same distribution as $Y^{\mathrm{o}}$ : (3.1) holds for $Y^{\mathrm{o}}$ if and only if it holds with $Y^{\mathrm{o}}$ replaced by this copy of $Y^{\mathrm{o}}$.) Put

$$
Y=\theta_{S} Y^{\mathrm{o}}
$$

and note that since $Y^{\mathrm{o}}$ is stationary and independent of $\left(\left(N^{\mathrm{o}}, X^{\mathrm{o}}\right), S\right)$, so is $Y$.

Since $Y$ is stationary and independent of $(N, X)$ we obtain from (4.3) that

$$
\mathrm{E}\left[f\left(\theta_{t}(N, X, Y)\right) \lambda\left(C_{0}\right)\right]=\mathrm{E}\left[f(N, X, Y) \lambda\left(C_{0}\right)\right], \quad f \in \mathscr{C} \otimes \mathscr{H} \otimes \mathscr{L}^{+}, t \in \mathbb{R}^{d} .
$$

In the same way as (4.7) followed from (4.3), we obtain that (4.8) implies

$$
\mathrm{E}\left[f\left(N^{\mathrm{o}}, X^{\mathrm{o}}, Y^{\mathrm{o}}\right)\right]=\mathrm{E}\left[\lambda\left(C_{0}\right) \sum_{p \in s(N) \cap[0,1)^{d}} f\left(\theta_{p}(N, X, Y)\right)\right], \quad f \in \mathscr{C} \otimes \mathscr{H} \otimes \mathscr{L}^{+} .
$$

Replace $f$ by $f\left(\theta_{\pi}(\cdot)\right)$ in (4.9) to obtain 


$$
\mathrm{E}\left[f\left(\theta_{\pi}\left(N^{\mathrm{o}}, X^{\mathrm{o}}, Y^{\mathrm{o}}\right)\right)\right]=\mathrm{E}\left[\lambda\left(C_{0}\right) \sum_{p \in s(N) \cap[0,1)^{d}} f\left(\theta_{\pi} \theta_{p}(N, X, Y)\right)\right] .
$$

Take $i \in \mathbb{Z}^{d}$ and note that

$$
\sum_{p \in s(N) \cap[0,1)^{d}} 1_{\left\{i \leqslant p+\pi\left(\theta_{p}(N, X, Y)\right)<i+1\right\}} f\left(\theta_{\pi} \theta_{p}(N, X, Y)\right)
$$

is obtained from $(N, X, Y)$ by the same function in $\mathscr{C} \otimes \mathscr{H} \otimes \mathscr{B}^{+}$as

$$
\sum_{p \in s(N) \cap[-i,-i+1)^{d}} 1_{\left\{0 \leqslant p+\pi\left(\theta_{p}(N, X, Y)\right)<1\right\}} f\left(\theta_{\pi} \theta_{p}(N, X, Y)\right)
$$

from $\theta_{-i}(N, X, Y)$. Applying (4.8), with $t$ replaced by $-i$, yields

$$
\begin{aligned}
& \mathrm{E}\left[\lambda\left(C_{0}\right) \sum_{p \in s(N) \cap[0,1)^{d}} 1_{\left\{i \leqslant p+\pi\left(\theta_{p}(N, X, Y)\right)<i+1\right\}} f\left(\theta_{\pi} \theta_{p}(N, X, Y)\right)\right] \\
& =\mathrm{E}\left[\lambda\left(C_{0}\right) \sum_{p \in s(N) \cap[-i,-i+1)^{d}} 1_{\left\{0 \leqslant p+\pi\left(\theta_{p}(N, X, Y)\right)<1\right\}} f\left(\theta_{\pi} \theta_{p}(N, X, Y)\right)\right] .
\end{aligned}
$$

Sum over $i \in \mathbb{Z}^{d}$ and compare with (4.10) to obtain

$$
\mathrm{E}\left[f\left(\theta_{\pi}\left(N^{\mathrm{o}}, X^{\mathrm{o}}, Y^{\mathrm{o}}\right)\right)\right]=\mathrm{E}\left[\lambda\left(C_{0}\right) \sum_{p \in S(N)} 1_{\left\{0 \leqslant p+\pi\left(\theta_{p}(N, X, Y)\right)<1\right\}} f\left(\theta_{\pi} \theta_{p}(N, X, Y)\right)\right] .
$$

Since $\theta_{\pi}$ is bijective we have that for each point $q \in s(N) \cap[0,1)^{d}$ there is a unique point $p \in s(N)$ such that $q=p+\pi\left(\theta_{p}(N, X, Y)\right)$. Applying this on the right-hand side yields (note that $\theta_{\pi} \theta_{p}(N, X, Y)=\theta_{p+\pi\left(\theta_{p}(N, X, Y)\right)}(N, X, Y)$ )

$$
\mathrm{E}\left[f\left(\theta_{\pi}\left(N^{\mathrm{o}}, X^{\mathrm{o}}, Y^{\mathrm{o}}\right)\right)\right]=\mathrm{E}\left[\lambda\left(C_{0}\right) \sum_{q \in s(N) \cap[0,1)^{d}} f\left(\theta_{q}(N, X, Y)\right)\right] .
$$

Compare this and (4.9) to obtain that

$$
\mathrm{E}\left[f\left(\theta_{\pi}\left(N^{\mathrm{o}}, X^{\mathrm{o}}, Y^{\mathrm{o}}\right)\right)\right]=\mathrm{E}\left[f\left(N^{\mathrm{o}}, X^{\mathrm{o}}, Y^{\mathrm{o}}\right)\right], \quad f \in \mathscr{M} \otimes \mathscr{H} \otimes \mathscr{B}^{+},
$$

holds for $Y^{\circ}$ and $\pi$ as in Definition 3.1. Thus (4.1) holds and the proof of Theorem 4.1 is complete.

\subsection{The backgrounds and point-maps in Section 3.6 suffice}

The family of background fields $Y^{\mathrm{o}}$ and point-maps $\kappa_{n}, n \in \mathbb{Z}$, defined in Section 3.6, depend on a parameter $0<h<\infty$ suppressed in the notation. This family played a crucial role in the above proof and does in fact suffice to characterize point-stationarity. Below (in the proof of Theorem 5.1) we shall need the following slightly more general result. 
Lemma 4.1. Let $V$ be a $[0,1)$-valued random variable which is independent of $\left(N^{\mathrm{o}}, X^{\mathrm{o}}\right)$ and the family of random fields $Y^{\mathrm{o}}$ in Section 3.6. Let $V^{\mathrm{o}}$ be the stationary random field defined by $V_{t}^{\mathrm{o}} \equiv V, t \in \mathbb{R}^{d}$. The pair $\left(N^{\mathrm{o}}, X^{\mathrm{o}}\right)$ is point-stationary if and only if

$$
\theta_{\Pi_{n}^{\mathrm{o}}}\left(N^{\mathrm{o}}, X^{\mathrm{o}}\right) \stackrel{D}{=}\left(N^{\mathrm{o}}, X^{\mathrm{o}}\right)
$$

for all random points $\Pi_{n}^{\mathrm{o}}$ of the form

$$
\Pi_{n}^{\mathrm{o}}=\alpha_{n}\left(N^{\mathrm{o}}, X^{\mathrm{o}}, Y^{\mathrm{o}}, V^{\mathrm{o}}\right)
$$

where $\alpha_{n}, n \in \mathbb{Z}$, are the $\left(N^{\mathrm{o}}, X^{\mathrm{o}}, Y^{\mathrm{o}}, V^{\mathrm{o}}\right)$-point-maps defined by

$$
\alpha_{n}(\mu, x, y, v)=\kappa_{\left[v_{0} k\right]+n}(\mu, y),
$$

with $k=\mu\left(y_{0}+[-h / 2, h / 2)^{d}\right)$ and $[\cdot]$ denoting the integer part.

Proof. The 'only if' part holds because $\left(Y^{\circ}, V^{\mathrm{o}}\right)$, regarded as a bivariate random field, $\left(Y_{t}^{\mathrm{o}}, V_{t}^{\mathrm{o}}\right)_{s \in \mathbb{R}^{d}}$, is stationary and independent of $\left(N^{\mathrm{o}}, X^{\mathrm{o}}\right)$ and $\theta_{\alpha_{n}}$ is bijective for the same reason as $\theta_{\kappa_{n}}$ is: see the end of Section 3.6 and replace the origin in that argument by $\alpha_{0}(\mu, x, y, v)$.

In order to establish the 'if' part, let $S$ be a random site such that the conditional distribution of $S$ given $\left(N^{\mathrm{o}}, X^{\mathrm{o}}\right)$ is uniform on $C_{0}^{\mathrm{o}}$. Replace $Y^{\mathrm{o}}$ by $\left(Y^{\mathrm{o}}, V^{\mathrm{o}}\right)$ and $\kappa_{n}$ by $\alpha_{n}$ in the argument in Section 4.4 to obtain that (4.3) holds. By Theorem 4.1 this implies that $\left(N^{\mathrm{o}}, X^{\mathrm{o}}\right)$ is point-stationary.

\subsection{Palm characterization of stationarity}

Modifying the proof of Theorem 4.1 in an obvious way yields the following dual result.

Theorem 4.2. Let $(N, X),\left(N^{\mathrm{o}}, X^{\mathrm{o}}\right)$ and $S$ be linked as in Section 4.2 and suppose $\lambda\left(C_{0}\right)<\infty$ with probability one. Then $(N, X)$ is stationary if and only if the conditional distribution of $S$ given $\left(N^{\mathrm{o}}, X^{\mathrm{o}}\right)$ is uniform on $C_{0}^{\mathrm{o}}$ and

$$
\mathrm{E}\left[f\left(\theta_{\Pi^{\circ}}\left(N^{\mathrm{o}}, X^{\mathrm{o}}\right)\right) / \lambda\left(C_{0}\right)\right]=\mathrm{E}\left[f\left(N^{\mathrm{o}}, X^{\mathrm{o}}\right) / \lambda\left(C_{0}\right)\right], \quad f \in \mathscr{C}^{\mathrm{o}} \otimes \mathscr{H}^{+},
$$

for all random points $\Pi^{\mathrm{o}}$ as in Definition 3.1.

Proof. Simply replace E[·] by E[·/ $\left.\lambda\left(C_{0}\right)\right]$ throughout the proof of Theorem 4.1 .

Thus stationarity of the pair $(N, X)$ means that if we view it from the point of the Voronoi cell where the origin lies then the origin is located uniformly at random in the cell and, moreover, the pair looks the same from all observation points provided we volume-debias by the volume of the cell.

We shall need the following result in Section 6 .

Lemma 4.2. If $(N, X)$ is stationary then 


$$
\mathrm{E}\left[f\left(N^{\mathrm{o}}, X^{\mathrm{o}}\right) / \lambda\left(C_{0}\right)\right]=\mathrm{E}\left[\sum_{p \in s(N) \cap[0,1)^{d}} f\left(\theta_{p}(N, X)\right)\right], \quad f \in \mathscr{L} \otimes \otimes \mathscr{H}^{+} .
$$

In particular,

$$
\mathrm{E}\left[1 / \lambda\left(C_{0}\right)\right]=\mathrm{E}\left[N\left([0,1)^{d}\right)\right]
$$

Proof. In the proof of (4.7) replace $\mathrm{E}[\cdot]$ by $\mathrm{E}\left[\cdot / \lambda\left(C_{0}\right)\right]$ to obtain (4.12). Then take $f \equiv 1$ to obtain (4.13).

The expected number of points in a unit box, $\mathrm{E}\left[N\left([0,1)^{d}\right)\right]$, is called the intensity of the stationary point process $N$. According to (4.13) it can be calculated by taking the expectation of one over the volume of the Voronoi cell containing the origin, $\mathrm{E}\left[1 / \lambda\left(C_{0}\right)\right]$.

\section{Point-stationarity characterized by randomized point-shifts}

In this section we shall show that point-stationarity means distributional invariance under doubly randomized point-shifts: a shift to a uniformly selected site followed by a shift to a uniformly selected point.

\subsection{The characterization result}

It turns out that a point-stationary $\left(N^{\mathrm{o}}, X^{\mathrm{o}}\right)$ is characterized by the following property: if $\left(N^{\mathrm{o}}, X^{\mathrm{o}}\right)$ is first shifted by an independent site $U$ selected uniformly at random in any bounded Borel set $B$ of positive volume and the origin is then shifted to a $\theta_{-U} N^{\mathrm{o}}$-point $\Pi$ picked uniformly at random among the points $s\left(\theta_{-U} N^{\mathrm{o}}\right) \cap B$ that ended up in $B$, then the distribution of $\left(N^{\mathrm{o}}, X^{\mathrm{o}}\right)$ does not change. Note that there is at least one $\theta_{-U} N^{\mathrm{o}}$-point in $B$, the one at $U$ which initially was at the origin:

$$
\theta_{-U} N^{\mathrm{o}}(\{U\})=N^{\mathrm{o}}(\{0\})=1 .
$$

The first randomized shift would not change the distribution of a stationary pair: if $(N, X)$ is stationary then, since $U$ is independent of $(N, X)$,

$$
\theta_{-U}(N, X) \stackrel{D}{=}(N, X) \text {. }
$$

The first randomized shift does, however, change the distribution of a point-stationary pair since $\theta_{-U}\left(N^{\mathrm{o}}, X^{\mathrm{o}}\right)$ has no point at the origin, unlike $\left(N^{\mathrm{o}}, X^{\mathrm{o}}\right)$. But when the first randomized shift is followed by the second then the distribution is restored: if $\left(N^{\mathrm{o}}, X^{\mathrm{o}}\right)$ is pointstationary then

$$
\theta_{\Pi} \theta_{-U}\left(N^{\mathrm{o}}, X^{\mathrm{o}}\right) \stackrel{D}{=}\left(N^{\mathrm{o}}, X^{\mathrm{o}}\right) .
$$

Note that $\Pi^{\mathrm{o}}=\Pi-U$ is uniform on $s\left(N^{\mathrm{o}}\right) \cap(B-U)$ so we can describe this characteri- 
zation of a point-stationary $\left(N^{\mathrm{o}}, X^{\mathrm{o}}\right)$ alternatively as follows: if we place any bounded Borel set of positive volume uniformly at random around the $N^{\mathrm{o}}$-point at the origin and shift the origin to a point $\Pi^{\mathrm{o}}$ selected uniformly at random among the $N^{\mathrm{o}}$-points in that set then the distribution of $\left(N^{\mathrm{o}}, X^{\mathrm{o}}\right)$ does not change.

Here is a more formal statement.

Theorem 5.1. Let $B \in \mathscr{\beta}^{d}$ be bounded and such that $\lambda(B)>0$. Let $U$ be a random site that is uniform on $B$ and independent of $\left(N^{\mathrm{o}}, X^{\mathrm{o}}\right)$. Let $\Pi^{\mathrm{o}}$ be a random point of $N^{\mathrm{o}}$ such that the conditional distribution of $\Pi^{\mathrm{o}}$ given $\left(\left(N^{\mathrm{o}}, X^{\mathrm{o}}\right), U\right)$ is uniform on the finite set of points of $N^{\mathrm{o}}$ lying in $B-U$, that is, uniform on

$$
s\left(N^{\mathrm{o}}\right) \cap(B-U) .
$$

Then $\left(N^{\mathrm{o}}, X^{\mathrm{o}}\right)$ is point-stationary if and only if for each such $B$

$$
\left(\theta_{\Pi^{\mathrm{o}}}\left(N^{\mathrm{o}}, X^{\mathrm{o}}\right), \Pi^{\mathrm{o}}+U\right) \stackrel{D}{=}\left(\left(N^{\mathrm{o}}, X^{\mathrm{o}}\right), U\right) .
$$

In fact, $\left(N^{\mathrm{o}}, X^{\mathrm{o}}\right)$ is point-stationary if (5.1) holds for all $B$ of the form

$$
B=[-h / 2, h / 2)^{d}, \quad h>0 .
$$

Note that it follows from (5.1) that if we place a bounded Borel set of positive volume uniformly at random around the point at the origin then the point at the origin is located uniformly at random among the points in the set.

\subsection{Proof of Theorem 5.1}

Let $Y^{\circ}$ be as in Section 3.6 and choose $h$ large enough for $B$ to be contained in $[-h / 2, h / 2)^{d}$. Put

$$
B^{\prime}=[-h / 2, h / 2)^{d} \backslash B .
$$

Let $\beta_{n}, n \in \mathbb{Z}$, be the following modification of the point-shifts $\kappa_{n}$ in Section 3.6: if $0 \in y_{0}+B$ let $\beta_{n}(\mu, y)$ be the $n$th point after the point at the origin in the circular lexicographic enumeration of the $\mu$-points in $y_{0}+B$; if $0 \in y_{0}+B^{\prime}$ let $\beta_{n}(\mu, y)$ be the $n$th point after the point at the origin in the circular lexicographic enumeration of the $\mu$-points in $y_{0}+B^{\prime}$. Then $\theta_{\beta_{n}}$ is bijective for the same reason as $\theta_{\kappa_{n}}$ is bijective (see Section 3.6).

Note that conditionally on $-Y_{0}^{\mathrm{o}} \in B, Y_{0}^{\mathrm{o}}+B$ is $B$ placed uniformly at random around the origin. In order to obtain a point placed uniformly at random among the points in $Y_{0}^{\mathrm{o}}+B$, let $V$ be uniform of $[0,1)$ and independent of $\left(N^{\mathrm{o}}, X^{\mathrm{o}}, Y^{\mathrm{o}}\right)$, and define a stationary random field $V^{\mathrm{o}}$ by

$$
V_{t}^{\mathrm{o}} \equiv V, \quad t \in \mathbb{R}^{d} .
$$

For each $n \in \mathbb{Z}$, define $\left(N^{\mathrm{o}}, X^{\mathrm{o}}, Y^{\mathrm{o}}, V^{\mathrm{o}}\right)$-point-maps $\alpha_{n}$ as follows: if $0 \in y_{0}+B$ let $\alpha_{n}(\mu, x, y, v)=\beta_{\left[v_{0} k\right]+n}(\mu, y)$, where $k=\mu\left(y_{0}+B\right)$, and if $0 \in y_{0}+B^{\prime}$ let $\alpha_{n}(\mu, x, y, v)=$ 
$\beta_{n}(\mu, y)$. Then $\theta_{\alpha_{n}}$ is bijective and $\left(Y^{\mathrm{o}}, V^{\mathrm{o}}\right)$, regarded as a bivariate random field, $\left(Y_{t}^{\mathrm{o}}, V_{t}^{\mathrm{o}}\right)_{s \in \mathbb{R}^{d}}$, is stationary and independent of $\left(N^{\mathrm{o}}, X^{\mathrm{o}}\right)$.

With

$$
\Pi_{n}^{\mathrm{o}}=\alpha_{n}\left(N^{\mathrm{o}}, X^{\mathrm{o}}, Y^{\mathrm{o}}, V^{\mathrm{o}}\right)
$$

we have

$$
P\left(\left(\left(N^{\mathrm{o}}, X^{\mathrm{o}}\right), \Pi_{n}^{\mathrm{o}}-Y_{0}^{\mathrm{o}},-Y_{0}^{\mathrm{o}}\right) \in \cdot \mid-Y_{0}^{\mathrm{o}} \in B\right)=P\left(\left(\left(N^{\mathrm{o}}, X^{\mathrm{o}}\right), \Pi^{\mathrm{o}}+U, U\right) \in \cdot\right) .
$$

Suppose $\left(N^{\mathrm{o}}, X^{\mathrm{o}}\right)$ is point-stationary. Then

$$
\theta_{\Pi_{n}^{\mathrm{o}}}\left(N^{\mathrm{o}}, X^{\mathrm{o}}, Y^{\mathrm{o}}, V^{\mathrm{o}}\right) \stackrel{D}{=}\left(N^{\mathrm{o}}, X^{\mathrm{o}}, Y^{\mathrm{o}}, V^{\mathrm{o}}\right),
$$

which implies

$$
\left(\theta_{\Pi_{n}^{\mathrm{o}}}\left(N^{\mathrm{o}}, X^{\mathrm{o}}\right),-Y_{\Pi_{n}^{\mathrm{o}}}^{\mathrm{o}}\right) \stackrel{D}{=}\left(\left(N^{\mathrm{o}}, X^{\mathrm{o}}\right),-Y_{0}^{\mathrm{o}}\right)
$$

Now

$$
\left\{-Y_{0}^{\mathrm{o}} \in B\right\}=\left\{-Y_{\Pi_{n}^{0}}^{\mathrm{o}} \in B\right\}, \quad-Y_{\Pi_{n}^{\mathrm{o}}}^{\mathrm{o}}=\Pi_{n}^{\mathrm{o}}-Y_{0}^{\mathrm{o}},
$$

which yields the second identity in

$$
\begin{array}{rlrl}
P\left(\left(\theta_{\Pi^{\mathrm{o}}}\left(N^{\mathrm{o}}, X^{\mathrm{o}}\right), \Pi^{\mathrm{o}}+U\right)\right. & \in \cdot) & \\
& =P\left(\left(\theta_{\Pi_{n}^{0}}\left(N^{\mathrm{o}}, X^{\mathrm{o}}\right), \Pi_{n}^{\mathrm{o}}-Y_{0}^{\mathrm{o}}\right) \in \cdot \mid-Y_{0}^{\mathrm{o}} \in B\right) & & \text { (due to (5.2)) } \\
& =P\left(\left(\theta_{\Pi_{n}^{\mathrm{o}}}\left(N^{\mathrm{o}}, X^{\mathrm{o}}\right),-Y_{\Pi_{n}^{\mathrm{o}}}^{\mathrm{o}}\right) \in \cdot \mid-Y_{\Pi_{n}^{\mathrm{o}}}^{\mathrm{o}} \in B\right) & & \text { (due to (5.4)) } \\
& =P\left(\left(\left(N^{\mathrm{o}}, X^{\mathrm{o}}\right),-Y_{0}^{\mathrm{o}}\right) \in \cdot \mid-Y_{0}^{\mathrm{o}} \in B\right) & & \text { (due to (5.3)) } \\
& =P\left(\left(\left(N^{\mathrm{o}}, X^{\mathrm{o}}\right), U\right) \in \cdot\right) & & \text { (due to (5.2)). }
\end{array}
$$

Thus point-stationarity implies (5.1) for all bounded $B$ of positive Lebesgue measure.

Conversely, suppose (5.1) holds for $B=[-h / 2, h / 2)^{d}$. With this $B(5.2)$ becomes

$$
P\left(\left(\left(N^{\mathrm{o}}, X^{\mathrm{o}}\right), \Pi_{n}^{\mathrm{o}}-Y_{0}^{\mathrm{o}},-Y_{0}^{\mathrm{o}}\right) \in \cdot\right)=P\left(\left(\left(N^{\mathrm{o}}, X^{\mathrm{o}}\right), \Pi^{\mathrm{o}}+U, U\right) \in \cdot\right),
$$

which implies $\theta_{\Pi_{n}^{\circ}}\left(N^{\mathrm{o}}, X^{\mathrm{o}}\right) \stackrel{D}{=} \theta_{\Pi^{\circ}}\left(N^{\mathrm{o}}, X^{\mathrm{o}}\right)$. Thus, due to $(5.1)$,

$$
\theta_{\Pi_{n}^{\mathrm{o}}}\left(N^{\mathrm{o}}, X^{\mathrm{o}}\right) \stackrel{D}{=}\left(N^{\mathrm{o}}, X^{\mathrm{o}}\right)
$$

that is, (4.11) in Lemma 4.1 holds. Also, when $B=[-h / 2, h / 2)^{d}$ the $\alpha_{n}$ satisfy the condition in Lemma 4.1. Thus $\left(N^{\mathrm{o}}, X^{\mathrm{o}}\right)$ is point-stationary.

\section{Point-stationarity and the invariant $\sigma$-algebras $\mathscr{T}$ and}

In this section we first define the invariant $\sigma$-algebras $\mathscr{T}$ and $\mathscr{J}$, then extend Theorem 5.1 
slightly, and finally show that several properties are preserved under conditioning on $\mathscr{J}$, the invariant $\sigma$-algebra of both $(N, X)$ and $\left(N^{\mathrm{o}}, X^{\mathrm{o}}\right) \equiv \theta_{\Pi_{0}}(N, X)$.

\subsection{The invariant $\sigma$-algebras $\mathscr{T}$ and $\mathscr{T}$}

Let $\mathscr{T}$ be the invariant $\sigma$-algebra on $\mathscr{l} \otimes \mathscr{H}$, namely the following sub- $\sigma$-algebra of $\mathscr{l b} \otimes \mathscr{H}:$

$$
\mathscr{T}=\left\{B \in \mathscr{l} b \otimes \mathscr{H}: \theta_{t} B=B, t \in \mathbb{R}^{d}\right\} .
$$

Let $\mathscr{J}$ be the invariant $\sigma$-algebra of $(N, X)$, namely the following sub- $\sigma$-algebra of $\mathscr{F}$ :

$$
\mathscr{J}=(N, X)^{-1} \mathscr{T},
$$

(short for $\mathscr{J}=\{\{(N, X) \in B\}: B \in \mathscr{T}\})$. Note that, for any random site $T$ and any $B \in \mathscr{T}$,

$$
\begin{aligned}
\left\{\theta_{t}(N, X) \in B, T=t\right\} & =\left\{(N, X) \in \theta_{-t} B, T=t\right\} \\
& =\{(N, X) \in B, T=t\}, \quad t \in \mathbb{R}^{d},
\end{aligned}
$$

and thus taking union over $t \in \mathbb{R}^{d}$ yields

$$
\left\{\theta_{T}(N, X) \in B\right\}=\{(N, X) \in B\}, \quad B \in \mathscr{T} .
$$

In particular, with $\left(N^{\mathrm{o}}, X^{\mathrm{o}}\right) \equiv \theta_{\Pi_{0}}(N, X)$ this yields

$$
\mathscr{J}=\left(N^{\mathrm{o}}, X^{\mathrm{o}}\right)^{-1} \mathscr{T},
$$

that is, when $(N, X)$ and $\left(N^{\mathrm{o}}, X^{\mathrm{o}}\right)$ are linked as in Section 4.2 then $\mathscr{J}$ is also the invariant $\sigma$ algebra of $\left(N^{\mathrm{o}}, X^{\mathrm{o}}\right)$.

Note that although we have chosen to regard $N^{\mathrm{o}}$ as a random element in $\left(M^{\mathrm{o}}, \mathscr{C l}^{\mathrm{o}}\right)$, and not in $(M, \mathscr{C})$, the invariant $\sigma$-algebra of $\left(N^{\mathrm{o}}, X^{\mathrm{o}}\right) \equiv \theta_{\Pi_{0}}(N, X)$ is still $\mathscr{J}$ since

$$
\mathscr{T}^{\mathrm{o}}=\text { the invariant } \sigma \text {-algebra on }\left(M^{\mathrm{o}}, \mathscr{C l}^{\mathrm{o}}\right)=\text { the trace of } M^{\mathrm{o}} \times H \text { on } \mathscr{T}
$$

and thus $\mathscr{J}=\left(N^{\mathrm{o}}, X^{\mathrm{o}}\right)^{-1} \mathscr{T}=\left(N^{\mathrm{o}}, X^{\mathrm{o}}\right)^{-1} \mathscr{T}^{\mathrm{o}}$.

\subsection{Extension of Theorem 5.1}

We shall now extend Theorem 5.1 by allowing the set $B$ to be expanded by an invariant random variable. This result will be used in the proof of Theorem 9.3.

Theorem 6.1. Let $g \in \mathscr{T}$ be a strictly positive and finite function and put

$$
G \equiv g\left(N^{\mathrm{o}}, X^{\mathrm{o}}\right)
$$

Let $B \in \mathscr{B}^{d}$ be bounded and such that $\lambda(B)>0$. Let $U$ be a random site that is uniform on $B$ and independent of $\left(N^{\mathrm{o}}, X^{\mathrm{o}}\right)$. Let $\Pi^{\mathrm{o}}$ be a random point of $N^{\mathrm{o}}$ such that the conditional distribution of $\Pi^{\mathrm{o}}$ given $\left(\left(N^{\mathrm{o}}, X^{\mathrm{o}}\right), U\right)$ is uniform on the finite set of points of $N^{\mathrm{o}}$ lying in $G(B-U)$; that is, let $\Pi^{\mathrm{o}}$ be uniform on 


$$
s\left(N^{\mathrm{o}}\right) \cap G(B-U) .
$$

Then $\left(N^{\mathrm{o}}, X^{\mathrm{o}}\right)$ is point-stationary if and only if for each such $B$

$$
\left(\theta_{\Pi^{\circ}}\left(N^{\mathrm{o}}, X^{\mathrm{o}}\right), \Pi^{\mathrm{o}}+G U\right) \stackrel{D}{=}\left(\left(N^{\mathrm{o}}, X^{\mathrm{o}}\right), G U\right) .
$$

Proof. First assume that $g$ is bounded and repeat the proof of Theorem 5.1 with $B$ replaced by $g(\mu, x) B$. Now $g \in \mathscr{T}$ means that $g\left(\theta_{t}(\mu, x)\right) B=g(\mu, x) B$ for all $t \in \mathbb{R}^{d}$. This fact - that the set $g(\mu, x) B$ does not change by shifting $(\mu, x)-$ is needed to deduce that $\theta_{\beta_{n}}$ is still bijective. Thus (6.4) holds for bounded $g$. If $g$ is not bounded, take a finite constant $a>0$ and apply (6.4), with $g$ replaced by $g \wedge a$, to obtain

$$
\begin{aligned}
& \mathrm{E}\left[f \left(\theta_{\Pi^{\circ}}\left(N^{\mathrm{o}}, X^{\mathrm{o}}\right), \Pi^{\mathrm{o}}+\right.\right.\left.G U) 1\left\{g\left(\theta_{\Pi^{\mathrm{o}}}\left(N^{\mathrm{o}}, X^{\mathrm{o}}\right)\right)<a\right\}\right] \\
&=\mathrm{E}\left[f\left(\left(N^{\mathrm{o}}, X^{\mathrm{o}}\right), G U\right) 1\left\{g\left(N^{\mathrm{o}}, X^{\mathrm{o}}\right)<a\right\}\right], \quad f \in \mathscr{K} \otimes \mathscr{H} \otimes \mathscr{B}^{d^{+}} .
\end{aligned}
$$

Send $a$ to infinity to obtain (6.4).

\subsection{Conditioning on the invariant $\sigma$-algebra}

We shall now show that all characterizations still hold when we condition on the invariant $\sigma-$ algebra $\mathscr{J}$ of $(N, X)$ and of $\left(N^{\mathrm{o}}, X^{\mathrm{o}}\right) \equiv \theta_{\Pi_{0}}(N, X)$. This result will be used in Section 9.

Theorem 6.2. Let $\left(N^{\mathrm{o}}, X^{\mathrm{o}}\right), S$ and $(N, X)$ be linked as in Section 4.2.

(a) The pair $\left(N^{\mathrm{o}}, X^{\mathrm{o}}\right)$ is point-stationary under $P$ if and only if it is so under $P(\cdot \mid \mathscr{J})$.

(b) The pair $(N, X)$ is stationary under $P$ if and only if it is so under $P(\cdot \mid \mathscr{J})$.

(c) Formula (4.3) holds if and only if it holds with E replaced by $\mathrm{E}[\cdot \mid \mathscr{J}]$.

(d) Formulae (5.1) and (6.4) hold if and only if they hold when $P$ is replaced by $P(\cdot \mid \mathscr{J})$.

(e) If $(N, X)$ is stationary under $P$ then

$$
\mathrm{E}\left[N\left([0,1)^{d}\right) \mid \mathscr{J}\right]=\mathrm{E}\left[1 / \lambda\left(C_{0}\right) \mid \mathscr{Z}\right] \text { a.s. } P .
$$

Proof. Formula (3.1) in the definition of point-stationarity is equivalent to

$$
\begin{aligned}
& \mathrm{E}\left[f\left(\theta_{\Pi^{\mathrm{o}}}\left(N^{\mathrm{o}}, X^{\mathrm{o}}, Y^{\mathrm{o}}\right)\right) 1_{\left\{\theta_{\left.\Pi^{\mathrm{o}}\left(N^{\mathrm{o}}, X^{\mathrm{o}}\right) \in B\right\}}\right]}\right. \\
& \mathrm{E}\left[f\left(N^{\mathrm{o}}, X^{\mathrm{o}}, Y^{\mathrm{o}}\right) 1_{\left\{\left(N^{\mathrm{o}}, X^{\mathrm{o}}\right) \in B\right\}}\right], \quad B \in \mathscr{T}, f \in \mathscr{C} \otimes \mathscr{H}^{+} .
\end{aligned}
$$

Due to (6.2), we have that

$$
1_{\left\{\theta_{\Pi^{\circ}}\left(N^{\circ}, X^{\circ}\right) \in B\right\}}=1_{\left\{\left(N^{\circ}, X^{\circ}\right) \in B\right\}}, \quad B \in \mathscr{T},
$$

and thus (3.1) is equivalent to

$$
\mathrm{E}\left[f\left(\theta_{\Pi^{\circ}}\left(N^{\mathrm{o}}, X^{\mathrm{o}}, Y^{\mathrm{o}}\right)\right) \mid \mathscr{J}\right]=\mathrm{E}\left[f\left(N^{\mathrm{o}}, X^{\mathrm{o}}, Y^{\mathrm{o}}\right) \mid \mathscr{J}\right], \quad f \in \mathscr{K} \otimes \mathscr{H}^{+} .
$$

This yields (a). We obtain (b), (c) and (d) in a similar way. 
In order to obtain (e), take $f \in \mathscr{T}$ in Lemma 4.2. Then $f\left(\theta_{p}(N, X)\right)=f\left(N^{\mathrm{o}}, X^{\mathrm{o}}\right)$ and we obtain from (4.12) that

$$
\mathrm{E}\left[f\left(N^{\mathrm{o}}, X^{\mathrm{o}}\right) / \lambda\left(C_{0}\right)\right]=\mathrm{E}\left[f\left(N^{\mathrm{o}}, X^{\mathrm{o}}\right) N\left([0,1)^{d}\right)\right], \quad f \in \mathscr{T},
$$

that is, (e) holds.

\section{On Palm theory of stationary point processes}

We are now ready to extend to $d>1$ dimensions the approach to Palm theory presented in Thorisson (1995) for $d=1$. Since this approach differs from the conventional one, let us first have a brief look at the conventional theory.

\subsection{On the conventional Palm theory of stationary point processes}

The so-called Palm version $\left(N^{\mathrm{o}}, X^{\mathrm{o}}\right)$ of a stationary pair $(N, X)$ having finite intensity:

$$
\mathrm{E}\left[1 / \lambda\left(C_{0}\right)\right]=\mathrm{E}\left[N\left([0,1)^{d}\right)\right]<\infty
$$

is usually defined by specifying the expectations of $f\left(N^{\mathrm{o}}, X^{\mathrm{o}}\right)$ to coincide with

$$
\mathrm{E}\left[\sum_{p \in s(N) \cap[0,1)^{d}} f\left(\theta_{p}(N, X)\right)\right] / \mathrm{E}\left[N\left([0,1)^{d}\right)\right], \quad f \in \mathscr{C} \otimes \mathscr{H}^{+}
$$

(compare with (4.12) and Theorem 4.2). This $\left(N^{\mathrm{o}}, X^{\mathrm{o}}\right)$ has the following interpretation: $\left(N^{\mathrm{o}}, X^{\mathrm{o}}\right)$ behaves as $(N, X)$ when $N$ happens to have a point at the origin. (That is, the distribution of $\left(N^{\mathrm{o}}, X^{\mathrm{o}}\right)$ is the conditional distribution of $(N, X)$ when it is given that $N$ has a point at the origin. Since the probability of $N$ having a point at the origin is zero this interpretation is informal and must be motivated, for instance, by a limit theorem.)

The distribution of the Palm version $\left(N^{\mathrm{o}}, X^{\mathrm{o}}\right)$ can be reversed to get back the distribution of the stationary pair $(N, X)$. Thus there is a duality (one-to-one correspondence) between the distributions of stationary pairs $(N, X)$ with finite intensity and their Palm versions $\left(N^{\mathrm{o}}, X^{\mathrm{o}}\right)$. This is not a very satisfying duality since the Palm version is derived from the stationary $(N, X)$. In other words, the Palm version does not have a defining property without reference to its stationary dual and thus the duality is rather halfbaked.

When $d=1$, however, it is known that the Palm version $\left(N^{\mathrm{o}}, X^{\mathrm{o}}\right)$ is cycle-stationary (that is, point-stationary) with cycle lengths having finite mean. Moreover, it is known that the reversed Palm of any cycle-stationary $\left(N^{\mathrm{o}}, X^{\mathrm{o}}\right)$ with cycle lengths having finite mean yields a stationary pair $(N, X)$ with finite intensity. Thus, when $d=1$, there is a Palm duality between stationary $(N, X)$ with finite intensity and point-stationary $\left(N^{\mathrm{o}}, X^{\mathrm{o}}\right)$ with cycle lengths having finite mean.

When $d>1$, there should be a Palm duality between two classes of processes of equal status: stationary and point-stationary. 


\subsection{The approach of this paper}

In this paper we have given meaning to point-stationarity when $d>1$. The task now is to extend the above-mentioned Palm duality to $d>1$. We shall do so along the lines of Thorisson (1995). This approach differs from the conventional one in two ways.

On the one hand, rather than defining the distribution of the Palm version by the single formula (7.1) (and then getting the stationary process back through a single reversion formula) we define the Palm duality in two separate steps. The first step is measure-free, involving only the paths (shifting to or from a point; Section 4.2). The other is a biasing step involving the probability measure and the volume of the Voronoi cell containing the origin. (Other expressions for biasing are weighting, Radon-Nikodym, change of measure.)

On the other hand, we present two Palm dualities rather than only one. These dualities have quite different interpretations:

(1) The first duality has the conventional informal interpretation that the point-stationary pair behaves like its stationary dual conditioned on having a point at the origin. In particular, this means that the point-stationary pair in this duality is the conventional Palm version of the stationary pair.

(2) The second duality has the informal interpretation that the point-stationary pair behaves like its stationary dual observed from a point selected uniformly at random among all the points. Conversely, the stationary pair behaves like its point-stationary dual observed from a site selected uniformly at random among all sites in $\mathbb{R}^{d}$.

In other words:

(1) If we watch a stationary pair $(N, X)$ from the origin and there happens to be a point there then $(N, X)$ behaves as its first point-stationary dual.

(2) If we watch a stationary pair $(N, X)$ from a uniformly selected point then $(N, X)$ behaves as its second point-stationary dual.

The two dualities coincide in the ergodic case (when the invariant $\sigma$-algebra is trivial). The exact condition for coincidence is more general; see Section 10.1.

We shall present these two Palm dualities in the next two sections. The two dualities are indicated by subscripts on the probability measures.

\section{The first Palm duality}

We start by extending from $d=1$ to $d>1$ the conventional (point-at-zero) Palm duality between stationarity and point-stationarity.

\subsection{The first Palm duality theorem}

Let $(N, X)$ and $\left(\left(N^{\mathrm{o}}, X^{\mathrm{o}}\right), S\right)$ be defined on the same measurable space $(\Omega, \mathscr{F})$ and linked as in Section 4.2, that is to say, 


$$
\left(N^{\mathrm{o}}, X^{\mathrm{o}}\right) \equiv \theta_{\Pi_{0}}(N, X) \quad \text { and } \quad S \equiv-\Pi_{0}
$$

or equivalently

$$
(N, X) \equiv \theta_{S}\left(N^{\mathrm{o}}, X^{\mathrm{o}}\right)
$$

Recall that $C_{0}$ and $C_{0}^{\mathrm{o}}$, respectively, are the Voronoi cells of $(N, X)$ and $\left(N^{\mathrm{o}}, X^{\mathrm{o}}\right)$ containing the origin and that they have the same Lebesgue measure $\lambda\left(C_{0}^{o}\right) \equiv \lambda\left(C_{0}\right)$.

Let $P_{1}$ and $P_{1}^{\mathrm{o}}$ be two probability measures on $(\Omega, \mathscr{F})$ satisfying

$$
\mathrm{E}_{1}\left[1 / \lambda\left(C_{0}\right)\right]<\infty
$$

and

$$
\left.\mathrm{d} P_{1}^{\mathrm{o}}=\frac{1}{\lambda\left(C_{0}\right) \mathrm{E}_{1}\left[1 / \lambda\left(C_{0}\right)\right]} \mathrm{d} P_{1} \quad \text { (volume-debiasing } P_{1}\right)
$$

or, equivalently,

$$
\mathrm{E}_{1}^{\mathrm{o}}\left[\lambda\left(C_{0}\right)\right]<\infty
$$

and

$$
\left.\mathrm{d} P_{1}=\frac{\lambda\left(C_{0}\right)}{\mathrm{E}_{1}^{\mathrm{o}}\left[\lambda\left(C_{0}\right)\right]} \mathrm{d} P_{1}^{\mathrm{o}} \quad \text { (volume-biasing } P_{1}^{\mathrm{o}}\right) .
$$

Note that

$$
\mathrm{E}_{1}\left[1 / \lambda\left(C_{0}\right)\right]=\frac{1}{\mathrm{E}_{1}^{\mathrm{o}}\left[\lambda\left(C_{0}\right)\right]}
$$

and that the conditional distribution of $S$ given $\left(N^{\mathrm{o}}, X^{\mathrm{o}}\right)$ is the same under $P_{1}$ as under $P_{1}^{\mathrm{o}}$ [since $\lambda\left(C_{0}\right)$ is determined by $\left(N^{\mathrm{o}}, X^{\mathrm{o}}\right)$ ].

Theorem 8.1. We have that

$$
(N, X) \text { is stationary under } P_{1}
$$

if and only if

$\left(N^{\mathrm{o}}, X^{\mathrm{o}}\right)$ is point-stationary under $P_{1}^{\mathrm{o}}$

and the conditional distribution of $S$ given $\left(N^{\mathrm{o}}, X^{\mathrm{o}}\right)$ is uniform on $C_{0}^{\mathrm{o}}$.

Proof. This is an immediate consequence of Theorem 4.1.

\subsection{Interpretation and properties}

Theorem 8.1 yields a duality between the distributions of stationary $(N, X)$ with finite intensity and point-stationary $\left(N^{\mathrm{o}}, X^{\mathrm{o}}\right)$ with finite-mean Voronoi cell volume, as follows. If the distribution of the stationary $(N, X)$ is given we obtain the distribution of a pointstationary $\left(N^{\mathrm{o}}, X^{\mathrm{o}}\right)$ immediately from the theorem. If, on the other hand, the distribution of 
the point-stationary $\left(N^{\mathrm{o}}, X^{\mathrm{o}}\right)$ is given then we can introduce a random site $S$ with a uniform conditional distribution on $C_{0}^{\mathrm{o}}$, given $\left(N^{\mathrm{o}}, X^{\mathrm{o}}\right)$, to obtain a distribution of a stationary $(N, X)$.

The following theorem yields an unusually strong limit motivation of the conventional point-at-zero interpretation of this duality. Put $\Pi_{0}=t=0$ in (c) to obtain the informal expression:

$$
P_{1}\left((N, X) \in \cdot \mid \Pi_{0}=0\right)=P_{1}^{\mathrm{o}}\left(\left(N^{\mathrm{o}}, X^{\mathrm{o}}\right) \in \cdot\right)
$$

(This expression is informal because $P_{1}\left(\Pi_{0}=0\right)=0$.)

Theorem 8.2. Suppose that the conditional distribution of $S$ given $\left(N^{\mathrm{o}}, X^{\mathrm{o}}\right)$ is uniform on $C_{0}^{\mathrm{o}}$ under $P_{1}$ (or, equivalently, under $P_{1}^{\mathrm{o}}$ ). Then the following claims hold:

(a) Under $P_{1}$ the random site $S$ is continuous with density

$$
\frac{P_{1}(S \in d s)}{d s}=\frac{P_{1}^{\mathrm{o}}\left(s \in C_{0}^{\mathrm{o}}\right)}{\mathrm{E}_{1}^{\mathrm{o}}\left[\lambda\left(C_{0}\right)\right]}, \quad s \in \mathbb{R}^{d} .
$$

(b) For each $A \in \mathscr{l} \otimes \otimes \mathscr{H}$,

$$
P_{1}\left(\left(N^{\mathrm{o}}, X^{\mathrm{o}}\right) \in A \mid S=s\right)=P_{1}^{\mathrm{o}}\left(\left(N^{\mathrm{o}}, X^{\mathrm{o}}\right) \in A \mid s \in C_{0}^{\mathrm{o}}\right), \quad s \in \mathbb{R}^{d} .
$$

(c) For each $A \in \mathscr{C} \otimes \mathscr{H}$, there is a version of $P_{1}\left(\theta_{\Pi_{0}}(N, X) \in A \mid \Pi_{0}=\cdot\right)$ such that

$$
P_{1}\left(\theta_{\Pi_{0}}(N, X) \in A \mid \Pi_{0}=t\right) \rightarrow P_{1}^{\mathrm{o}}\left(\left(N^{\mathrm{o}}, X^{\mathrm{o}}\right) \in A\right), \quad|t| \downarrow 0 .
$$

Proof. (a) For $B \in \mathscr{B}^{d}$,

$$
\begin{aligned}
P_{1}(S \in B) & =\mathrm{E}_{1}\left[\mathrm{E}_{1}\left[1_{\{S \in B\}} \mid\left(N^{\mathrm{o}}, X^{\mathrm{o}}\right)\right]\right] \\
& =\mathrm{E}_{1}\left[\lambda\left(B \cap C_{0}^{\mathrm{o}}\right) / \lambda\left(C_{0}\right)\right] \\
& =\mathrm{E}_{1}^{\mathrm{o}}\left[\lambda\left(B \cap C_{0}^{\mathrm{o}}\right)\right] / \mathrm{E}_{1}^{\mathrm{o}}\left[\lambda\left(C_{0}\right)\right] \\
& =\int_{B} P_{1}^{\mathrm{o}}\left(s \in C_{0}^{\mathrm{o}}\right) \mathrm{d} s / \mathrm{E}_{1}^{\mathrm{o}}\left[\lambda\left(C_{0}\right)\right] .
\end{aligned}
$$

(b) With $f \in \mathscr{l} \mathbb{B} \otimes \mathscr{H}^{+}$put

$$
g_{f}(s)=\mathrm{E}_{1}^{\mathrm{o}}\left[f\left(N^{\mathrm{o}}, X^{\mathrm{o}}\right) \mid s \in C_{0}^{\mathrm{o}}\right], \quad s \in \mathbb{R}^{d} .
$$

Let $h \in \mathscr{B}^{d}$ be a non-negative function and apply $(a)$ for the first step in 


$$
\begin{aligned}
\mathrm{E}_{1}\left[h(S) g_{f}(S)\right] & =\int_{\mathbb{R}^{d}} h(S) \mathrm{E}_{1}^{\mathrm{o}}\left[f\left(N^{\mathrm{o}}, X^{\mathrm{o}}\right) \mid s \in C_{0}^{\mathrm{o}}\right] P_{1}^{\mathrm{o}}\left(s \in C_{0}^{\mathrm{o}}\right) / \mathrm{E}_{1}^{\mathrm{o}}\left[\lambda\left(C_{0}\right)\right] \mathrm{d} s \\
& =\mathrm{E}_{1}^{\mathrm{o}}\left[f\left(N^{\mathrm{o}}, X^{\mathrm{o}}\right) \int_{\mathbb{R}^{d}} h(s) 1_{\left\{s \in C_{0}^{\mathrm{o}}\right\}} \mathrm{d} s\right] / \mathrm{E}_{1}^{\mathrm{o}}\left[\lambda\left(C_{0}\right)\right] \\
& =\mathrm{E}_{1}\left[f\left(N^{\mathrm{o}}, X^{\mathrm{o}}\right) \int_{\mathbb{R}^{d}} h(s) 1_{\left\{s \in C_{0}^{\mathrm{o}}\right\}} \mathrm{d} s / \lambda\left(C_{0}\right)\right]
\end{aligned}
$$

Apply the conditional uniformity of $S$ to obtain the desired result on the following form:

$$
\mathrm{E}_{1}\left[h(S) g_{f}(S)\right]=\mathrm{E}_{1}\left[h(S) f\left(N^{\mathrm{o}}, X^{\mathrm{o}}\right)\right], \quad f \in \mathscr{N} \otimes \mathscr{H}^{+} .
$$

(c) This follows from (b) since $\Pi_{0}=-S$, and $1_{\left\{-t \in C_{0}^{o}\right\}} \rightarrow 1$ pointwise as $|t| \downarrow 0$.

\section{The second Palm duality}

We now extend the less known duality from $d=1$ to $d>1$. It is obtained by conditioning on the invariant $\sigma$-algebra $\mathscr{J}$ before biasing. Thus, in particular, the two dualities coincide in the ergodic case (when $\mathscr{J}$ is trivial).

\subsection{The second Palm duality theorem}

Again let $(N, X)$ and $\left(\left(N^{\mathrm{o}}, X^{\mathrm{o}}\right), S\right)$ be defined on the same measurable space $(\Omega, \mathscr{F})$ and linked as in Section 4.2, that is,

$$
\left(N^{\mathrm{o}}, X^{\mathrm{o}}\right) \equiv \theta_{\Pi_{0}}(N, X) \quad \text { and } \quad S \equiv-\Pi_{0}
$$

or equivalently

$$
(N, X) \equiv \theta_{S}\left(N^{\mathrm{o}}, X^{\mathrm{o}}\right)
$$

Recall that $C_{0}$ and $C_{0}^{\mathrm{o}}$, respectively, are the Voronoi cells of $(N, X)$ and $\left(N^{\mathrm{o}}, X^{\mathrm{o}}\right)$ containing the origin and that they have the same Lebesgue measure $\lambda\left(C_{0}^{o}\right) \equiv \lambda\left(C_{0}\right)$. Recall from Section 6.1 that $(N, X)$ and $\left(N^{\mathrm{o}}, X^{\mathrm{o}}\right)$ have the same invariant $\sigma$-algebra, namely

$$
\mathscr{J}=(N, X)^{-1} \mathscr{T}=\left(N^{\mathrm{o}}, X^{\mathrm{o}}\right)^{-1} \mathscr{T},
$$

where

$$
\mathscr{T}=\left\{B \in \mathscr{M} \otimes \mathscr{H}^{+}: \theta_{t} B=B, t \in \mathbb{R}^{d}\right\} .
$$

Let $P_{2}$ and $P_{2}^{o}$ be probability measures on $(\Omega, \mathscr{F})$ satisfying

$$
\mathrm{E}_{2}\left[1 / \lambda\left(C_{0}\right) \mid \mathscr{J}\right]<\infty \text { a.s. } P_{2}
$$

and 


$$
\left.\mathrm{d} P_{2}^{\mathrm{o}}=\frac{1}{\lambda\left(C_{0}\right) \mathrm{E}_{2}\left[1 / \lambda\left(C_{0}\right) \mid \mathscr{J}\right]} \mathrm{d} P_{2} \quad \text { (volume-debiasing } P_{2} \text { given } \mathscr{J}\right)
$$

or, equivalently,

$$
\mathrm{E}_{2}^{\mathrm{o}}\left[\lambda\left(C_{0}\right) \mid \mathscr{J}\right]<\infty \text { a.s. } P_{2}^{\mathrm{o}}
$$

and

$$
\left.\mathrm{d} P_{2}=\frac{\lambda\left(C_{0}\right)}{\mathrm{E}_{2}^{\mathrm{o}}\left[\lambda\left(C_{0}\right) \mid \mathscr{J}\right]} \mathrm{d} P_{2}^{\mathrm{o}} \quad \text { (volume-biasing } P_{2}^{\mathrm{o}} \text { given } \mathscr{J}\right)
$$

Note that

$$
\begin{gathered}
P_{2}=P_{2}^{\mathrm{o}} \text { on } \mathscr{J}, \quad P_{2}((N, X) \in \cdot)=P_{2}^{\mathrm{o}}\left(\left(N^{\mathrm{o}}, X^{\mathrm{o}}\right) \in \cdot\right) \text { on } \mathscr{T}, \\
\mathrm{E}_{2}^{\mathrm{o}}\left[V \lambda\left(C_{0}\right) \mid \mathscr{J}\right]=\frac{\mathrm{E}_{2}[V \mid \mathscr{J}]}{\mathrm{E}_{2}\left[1 / \lambda\left(C_{0}\right) \mid \mathscr{J}\right]}, \quad V \in \mathscr{F}^{+} .
\end{gathered}
$$

and that the conditional distribution of $S$ given $\left(N^{\mathrm{o}}, X^{\mathrm{o}}\right)$ is the same under $P_{2}$ as under $P_{2}^{\mathrm{o}}$ (since $\lambda\left(C_{0}\right)$ is determined by $\left(N^{\mathrm{o}}, X^{\mathrm{o}}\right)$ and since $\mathscr{J}$ is contained in $\left.\sigma\left\{\left(N^{\mathrm{o}}, X^{\mathrm{o}}\right)\right\}\right)$.

Theorem 9.1. We have that

$$
(N, X) \text { is stationary under } P_{2}
$$

if and only if

$$
\left(N^{\mathrm{o}}, X^{\mathrm{o}}\right) \text { is point-stationary under } P_{2}^{\mathrm{o}}
$$

and the conditional distribution of $S$ given $\left(N^{\mathrm{o}}, X^{\mathrm{o}}\right)$ is uniform on $C_{0}^{\mathrm{o}}$.

Proof. By Theorem 6.2(b), $(N, X)$ is stationary under $P_{2}$ if and only if

$$
\mathrm{E}_{2}\left[f\left(\theta_{t}(N, X)\right) \mid \mathscr{J}\right]=\mathrm{E}_{2}[f(N, X) \mid \mathscr{J}], \quad f \in \mathscr{M} \otimes \mathscr{H} \mathscr{C}^{+}, t \in \mathbb{R}^{d} .
$$

By (9.2) this is equivalent to

$$
\mathrm{E}_{2}^{\mathrm{o}}\left[f\left(\theta_{t}(N, X)\right) \lambda\left(C_{0}\right) \mid \mathscr{J}\right]=\mathrm{E}_{2}^{\mathrm{o}}\left[f(N, X) \lambda\left(C_{0}\right) \mid \mathscr{J}\right], \quad f \in \mathscr{C} \otimes \mathscr{H}^{+}, t \in \mathbb{R}^{d} .
$$

Combining this, Theorem $6.2(c)$ and Theorem 4.1 completes the proof.

\subsection{Shift-coupling the stationary and point-stationary duals}

Theorem 9.1 yields (in the same way as described at the beginning of Section 8.2) a duality between the distributions of stationary $(N, X)$ with finite intensity given $\mathscr{J}$ and pointstationary $\left(N^{\mathrm{o}}, X^{\mathrm{o}}\right)$ with finite-mean Voronoi cell volume given $\mathscr{J}$.

According to the following theorem the stationary and point-stationary duals in this second duality are really the same, only seen from different sites. 
Theorem 9.2. The probability space $\left(\Omega, \mathscr{F}, P_{2}\right)$ can be extended to support a random point $\Pi$ of $N$ such that

$$
\theta_{\Pi}(N, X) \text { under } P_{2} \stackrel{D}{=}\left(N^{\mathrm{o}}, X^{\mathrm{o}}\right) \text { under } P_{2}^{\mathrm{o}}
$$

and the probability space $\left(\Omega, \mathscr{F}, P_{2}^{\mathrm{o}}\right)$ can be extended to support a random site $T^{\mathrm{o}}$ such that

$$
\theta_{T^{\mathrm{o}}}\left(N^{\mathrm{o}}, X^{\mathrm{o}}\right) \text { under } P_{2}^{\mathrm{o}} \stackrel{D}{=}(N, X) \text { under } P_{2} \text {. }
$$

Proof. Due to (9.1), this is a consequence of Theorem 2 in Thorisson (1996).

\subsection{Interpretation}

The following theorem yields a limit motivation of the randomized-origin interpretations of the second duality. Put $h=\infty$ and $G \equiv 1$ in (a) to obtain the informal expression:

$$
P_{2}^{\mathrm{o}}\left(\theta_{\text {uniform site in } \mathbb{R}^{d}}\left(N^{\mathrm{o}}, X^{\mathrm{o}}\right) \in \cdot\right)=P_{2}((N, X) \in \cdot)
$$

and put $h=\infty$ and $G \equiv 1$ in (b), or $n=\infty$ in (c), to obtain the informal expression:

$$
P_{2}\left(\theta_{\text {uniform point of } N}(N, X) \in \cdot\right)=P_{2}^{\mathrm{o}}\left(\left(N^{\mathrm{o}}, X^{\mathrm{o}}\right) \in \cdot\right) \text {. }
$$

(These expressions are informal because there is neither a uniform distribution on $\mathbb{R}^{d}$ nor a uniform distribution on a countable set of points.)

Theorem 9.3. Suppose the equivalent conditions in Theorem 9.1 hold. Let $B_{h} \in \mathscr{B}^{d}$, $0<h<\infty$, be a family of sets satisfying $0<\lambda\left(B_{h}\right)<\infty$ and, for all $t \in \mathbb{R}^{d}$,

$$
\lambda\left(B_{h} \cap\left(t+B_{h}\right)\right) / \lambda\left(B_{h}\right) \rightarrow 1, \quad h \rightarrow \infty,
$$

(which holds, for example, when $B_{h}=h B_{1}$ ). Let $g \in \mathscr{T}$ be strictly positive and finite and put

$$
G \equiv g\left(N^{\mathrm{o}}, X^{\mathrm{o}}\right)
$$

Then the following limit results hold:

(a) Let $U_{h}$ be uniformly distributed on $B_{h}$ and independent of $\left(N^{\mathrm{o}}, X^{\mathrm{o}}\right)$ under $P_{2}^{\mathrm{o}}$. Then

$$
\theta_{G U_{h}}\left(N^{\mathrm{o}}, X^{\mathrm{o}}\right) \text { under } P_{2}^{\mathrm{o}} \rightarrow(N, X) \text { under } P_{2}
$$

in total variation as $h \rightarrow \infty$.

(b) Let each $B_{h}$ be bounded. Let $T_{h}$ be a random site such that under $P_{2}$ the conditional distribution of $T_{h}$ given $(N, X)$ is uniform on $s(N) \cap G B_{h}$ when $s(N) \cap G B_{h} \neq \varnothing$. Then

$$
\theta_{T_{h}}(N, X) \text { under } P_{2} \rightarrow\left(N^{\mathrm{o}}, X^{\mathrm{o}}\right) \text { under } P_{2}^{\mathrm{o}}
$$

in total variation as $h \rightarrow \infty$.

(c) Let the $B_{h}$ be convex and compact and increase continuously from $\{0\}$ to $\mathbb{R}^{d}$ as $h$ increases from 0 to $\infty$. Let $\Pi_{n}, n \geqslant 0$, be the points of $N$ enumerated in the order 
they are hit by $B_{h}$ as $h$ increases and lexicographically if two or more are hit simultaneously. Let $U$ be uniform on $[0,1)$ and independent of $(N, X)$ under $P_{2}$. Then

$$
\theta_{\Pi_{[U n]}}(N, X) \text { under } P_{2} \rightarrow\left(N^{\mathrm{o}}, X^{\mathrm{o}}\right) \text { under } P_{2}^{\mathrm{o}}
$$

in total variation as $n \rightarrow \infty$.

Proof. (a) Due to (9.1), this is a consequence of Theorem 2 in Thorisson (1996).

(b) Let $U_{h}$ be as in (a). Let $\Pi_{h}^{\mathrm{o}}$ be a random point of $N^{\mathrm{o}}$ such that the conditional distribution of $\Pi_{h}^{\mathrm{o}}$ given $\left(\left(N^{\mathrm{o}}, X^{\mathrm{o}}\right), U_{h}\right)$ is uniform on $s\left(N^{\mathrm{o}}\right) \cap G\left(B_{h}-U_{h}\right)$. Apply Theorem 6.1 to obtain that, under $\mathrm{P}_{2}^{\mathrm{o}}$,

$$
\left(\theta_{\Pi_{h}} \theta_{-G U_{h}}\left(N^{\mathrm{o}}, X^{\mathrm{o}}\right), \Pi_{h}\right) \stackrel{D}{=}\left(\left(N^{\mathrm{o}}, X^{\mathrm{o}}\right), G U_{h}\right),
$$

where

$$
\Pi_{h}=\Pi_{h}^{\mathrm{o}}+G U_{h} .
$$

The conditional distribution of $\Pi_{h}$ given $\left(\left(N^{\mathrm{o}}, X^{\mathrm{o}}\right), U_{h}\right)$ is uniform on $s\left(\theta_{-G U_{h}} N^{\mathrm{o}}\right) \cap G B_{h}$. This set is determined by $\theta_{-G U_{h}}\left(N^{\mathrm{o}}, X^{\mathrm{o}}\right)$ - since $g \in \mathscr{T}$ implies $G=g\left(\theta_{-G U_{h}}\left(N^{\mathrm{o}}, X^{\mathrm{o}}\right)\right)-$ and thus the conditional distribution of $\Pi_{h}$ given $\theta_{-G U_{h}}\left(N^{\mathrm{o}}, X^{\mathrm{o}}\right)$ is uniform on $s\left(\theta_{-G U_{h}} N^{\mathrm{o}}\right) \cap G B_{h}$. It follows that, a.s. $P_{2}^{\mathrm{o}}\left(\theta_{-G U_{h}}\left(N^{\mathrm{o}}, X^{\mathrm{o}}\right) \in \cdot\right)$, we have

$$
P_{2}\left(T_{h} \in \cdot \mid(N, X)=\cdot\right)=P_{2}^{\mathrm{o}}\left(\Pi_{h} \in \cdot \mid \theta_{-G U_{h}}\left(N^{\mathrm{o}}, X^{\mathrm{o}}\right)=\cdot\right) .
$$

Thus, with $\|\cdot\|$ denoting the total variation norm,

$$
\begin{aligned}
\| P_{2}\left(\theta_{T_{h}}(N, X)\right. & \in \cdot)-P_{2}^{\mathrm{o}}\left(\left(N^{\mathrm{o}}, X^{\mathrm{o}}\right) \in \cdot\right) \| \\
& =\left\|P_{2}\left(\theta_{T_{h}}(N, X) \in \cdot\right)-P_{2}^{\mathrm{o}}\left(\theta_{\Pi_{h}} \theta_{-G U_{h}}\left(N^{\mathrm{o}}, X^{\mathrm{o}}\right) \in \cdot\right)\right\| \quad \text { (due to (9.3)) } \\
& \left.\leqslant \| P_{2}\left((N, X), T_{h}\right) \in \cdot\right)-P_{2}^{\mathrm{o}}\left(\left(\theta_{-G U_{h}}\left(N^{\mathrm{o}}, X^{\mathrm{o}}\right), \Pi_{h}\right) \in \cdot\right) \| \\
& =\left\|P_{2}((N, X) \in \cdot)-P_{2}^{\mathrm{o}}\left(\theta_{-G U_{h}}\left(N^{\mathrm{o}}, X^{\mathrm{o}}\right) \in \cdot\right)\right\| \quad \quad \text { (due to (9.4)). }
\end{aligned}
$$

Applying (a) yields the desired result.

(c) Due to Theorem $6.2(\mathrm{e}), \mathrm{E}_{2}\left[N\left([0,1)^{d}\right) \mid \mathscr{Z}\right]=\mathrm{E}_{2}\left[1 / \lambda\left(C_{0}\right) \mid \mathscr{J}\right]$ and by assumption $\mathrm{E}_{2}\left[1 / \lambda\left(C_{0}\right) \mid \mathscr{J}\right]<\infty$. Put $G \equiv \mathrm{E}_{2}\left[N\left([0,1)^{d}\right) \mid \mathscr{J}\right]^{-1 / d}$. Then $N(G \cdot)$ is stationary and

$$
\mathrm{E}_{2}\left[N\left(G[0,1)^{d}\right) \mid \mathscr{J}\right]=G^{d} \mathrm{E}_{2}\left[N\left([0,1)^{d}\right) \mid \mathscr{J}\right]=1 \text { a.s. } P_{2} \text {. }
$$

Let $h(n)$ be such that $\lambda\left(B_{h(n)}\right)=n$ and apply Theorem 10.2.IV in Daley and Vere-Jones (1988) to $N(G \cdot)$ to obtain

$$
N\left(G B_{h(n)}\right) / n \rightarrow 1 \text { a.s. } P_{2}, \quad n \rightarrow \infty .
$$

Let $T_{h}$ be as in (b) with $T_{h}=0$ when $s(N) \cap G B_{h}=\varnothing$. Interpret

$$
0^{-1} \sum_{1}^{0} 1_{\left\{\theta_{\Pi_{k-1}}(N, X) \in \cdot\right\}}=1_{\{(N, X) \in \cdot\}}
$$


and note that, for $n, m \geqslant 1$ and $0 \leqslant a_{1} \leqslant 1,0 \leqslant a_{2} \leqslant 1, \ldots$,

$$
n^{-1} \sum_{1}^{n} a_{k}-m^{-1} \sum_{1}^{m} a_{k} \leqslant \begin{cases}n\left(n^{-1}-m^{-1}\right)=1-n / m, & n \leqslant m, \\ (n-m) n^{-1}=1-m / n, & m \leqslant n,\end{cases}
$$

to obtain (with $\wedge$ denoting minimum and $\vee$ maximum)

$$
\begin{aligned}
P_{2}\left(\theta_{\Pi_{[U n]}}(N, X) \in \cdot\right)- & P_{2}\left(\theta_{T_{h(n)}}(N, X) \in \cdot\right) \\
& =\mathrm{E}_{2}\left[n^{-1} \sum_{1}^{n} 1_{\left\{\theta_{\Pi_{k-1}}(N, X) \in \cdot\right\}}-N\left(G B_{h(n)}\right)^{-1} \sum_{1}^{N\left(G B_{h(n)}\right)} 1_{\left\{\theta_{\Pi_{k-1}}(N, X) \in \cdot\right\}}\right] \\
& \leqslant 1-\mathrm{E}_{2}\left[\frac{n \wedge N\left(G B_{h(n)}\right)}{n \vee N\left(G B_{h(n)}\right)}\right] .
\end{aligned}
$$

Thus, with $\|\cdot\|$ denoting the total variation norm,

$$
\left\|P_{2}\left(\theta_{\pi_{[U n]}}(N, X) \in \cdot\right)-P_{2}\left(\theta_{T_{h(n)}}(N, X) \in \cdot\right)\right\| \leqslant 2-2 \mathrm{E}_{2}\left[\frac{n \wedge N\left(G B_{h(n)}\right)}{n \vee N\left(G B_{h(n)}\right)}\right] .
$$

By (9.5) and bounded convergence the expectation goes to 1 as $n \rightarrow \infty$ and thus

$$
\left\|P_{2}\left(\theta_{\Pi_{[U n]}}(N, X) \in \cdot\right)-P_{2}\left(\theta_{T_{h(n)}}(N, X) \in \cdot\right)\right\| \rightarrow 0, \quad n \rightarrow \infty .
$$

This, together with (b), yields the final step in

$$
\begin{aligned}
\| P_{2}\left(\theta_{\Pi_{[U n]}}(N, X) \in \cdot\right)- & P_{2}^{\mathrm{o}}\left(\left(N^{\mathrm{o}}, X^{\mathrm{o}}\right) \in \cdot\right) \| \\
& \leqslant\left\|P_{2}\left(\theta_{\Pi_{[U n]}}(N, X) \in \cdot\right)-P_{2}\left(\theta_{T_{h(n)}}(N, X) \in \cdot\right)\right\| \\
& +\left\|P_{2}\left(\theta_{T_{h(n)}}(N, X) \in \cdot\right)-P_{2}^{\mathrm{o}}\left(\left(N^{\mathrm{o}}, X^{\mathrm{o}}\right) \in \cdot\right)\right\| \\
& \rightarrow 0, \quad n \rightarrow \infty .
\end{aligned}
$$

\section{Comments and discussion}

We conclude with comments on the two Palm dualities, on references, and on a possible extension of the point-stationarity concept to more general random phenomena.

\subsection{The exact condition for coincidence of the two Palm dualities}

When is standing at the origin of a stationary point pattern and happening to find a point there equivalent to standing at a point selected uniformly at random from the point pattern?

The two dualities coincide when

$$
P_{1}=P_{2} \Leftrightarrow P_{1}^{\mathrm{o}}=P_{2}^{\mathrm{o}} .
$$


This holds if and only if

$$
\mathrm{E}_{2}\left[1 / \lambda\left(C_{0}\right) \mid \mathscr{J}\right]=\mathrm{E}_{2}\left[1 / \lambda\left(C_{0}\right)\right] \text { a.s. } P_{2}
$$

and if and only if

$$
\mathrm{E}_{2}^{\mathrm{o}}\left[\lambda\left(C_{0}\right) \mid \mathscr{J}\right]=\mathrm{E}_{2}^{\mathrm{o}}\left[\lambda\left(C_{0}\right)\right] \text { a.s. } P_{2}^{\mathrm{o}} .
$$

In particular, this is true in the ergodic case (that is, when $\mathscr{J}$ is trivial, under either measure) which is a common assumption in the literature.

The first Palm version is well known and widely used, but the second is not so well known. From the point of view of applications the second version, however, is often (for instance when averaging over the points) more natural than the first, which suggests a partial explanation of the common ergodicity assumption.

\subsection{A random site change hides the gap between the two dualities}

We can make the two Palm dualities coincide by a simple random change of site scale (provided $X$ is measurable under change of site scale, as when it has a Polish state space and right-continuous paths with left-hand limits). Let $(N, X)$ be stationary under a probability measure $P$ with $\mathrm{E}\left[1 / \lambda\left(C_{0}\right)\right]<\infty$ and change the site scale by

$$
G \equiv \mathrm{E}\left[1 / \lambda\left(C_{0}\right) \mid \mathscr{J}\right]^{-1 / d}
$$

to obtain

$$
\left(N(G \cdot),\left(X_{G s}\right)_{s \in \mathbb{R}^{d}}\right) .
$$

This new pair is stationary and the Voronoi cell containing the origin is $C_{0} / G$. Noting that $\lambda\left(C_{0} / G\right)=\lambda\left(C_{0}\right) / G^{d}$ and $G \in \mathscr{J}^{+}$yields

$$
\mathrm{E}\left[1 / \lambda\left(C_{0} / G\right) \mid \mathscr{J}\right]=\mathrm{E}\left[1 / \lambda\left(C_{0}\right) \mid \mathscr{J}\right] G^{d}=1 .
$$

The invariant $\sigma$-algebra of $\left(N(G \cdot),\left(X_{G s}\right)_{s \in \mathbb{R}^{d}}\right)$ is contained in $\mathscr{J}$ and thus

$$
\mathrm{E}\left[1 / \lambda\left(C_{0} / G\right) \mid\left(N(G \cdot),\left(X_{G s}\right)_{s \in \mathbb{R}^{d}}\right)^{-1} \mathscr{T}\right]=1 .
$$

Since also $\mathrm{E}\left[1 / \lambda\left(C_{0} / G\right)\right]=1$, the coincidence condition holds (see (10.1)). Thus the two point-stationary Palm duals of $\left(N(G \cdot),\left(X_{G s}\right)_{s \in \mathbb{R}^{d}}\right)$ coincide, that is, $\left(N(G \cdot),\left(X_{G s}\right)_{s \in \mathbb{R}^{d}}\right)$ has only one point-stationary Palm dual.

Note that $\lambda\left(C_{0} / G\right)=\lambda\left(C_{0}\right) \mathrm{E}\left[1 / \lambda\left(C_{0}\right) \mid \mathscr{J}\right]$ and thus the change of measure used to obtain this common point-stationary dual of $\left(N(G \cdot),\left(X_{G s}\right)_{s \in \mathbb{R}^{d}}\right)$ is the same as the change of measure used to obtain the point-stationary randomized-origin dual of $(N, X)$. Thus this procedure preserves the randomized-origin duality and not the point-at-zero duality.

In fact, we lose the point-at-zero duality by this procedure: the point-at-zero duality merges with the randomized-origin duality through the change of site scale and does not reappear when we return to the original time scale after change of measure (as the randomized-origin duality does). Thus the change of site scale is not a way to bridge the gap between the two dualities, it only hides it. To bridge the gap we cannot avoid a change of measure. 


\subsection{On references}

For $d=1$, see Thorisson (1995) for references on the first Palm duality (the conventional point-at-zero duality). The author is aware of the following references on the second (the randomized-origin duality): Nawrotzki (1978) and the recent papers by Glynn and Sigman (1992), Nieuwenhuis (1994; 1998), Thorisson (1995), and Miyazawa et al. (1999). The second duality is highlighted in Sigman (1994).

For $d>1$, see Matthes et al. (1978), Daley and Vere-Jones (1988), Stoyan et al. (1987) and Cowan (1978) for references on the conventional (point-at-zero) Palm version of stationary point-processes. The author is not aware of references on the other (the randomized-origin) Palm version.

The only references (beyond the final comment in his own 1995 paper) that the author has seen where the point-stationarity problem is mentioned are Mandelbrot (1983) and Kagan and Vere-Jones (1988), which we discuss below.

\subsection{Extending point-stationarity to more general random sets?}

When working on the revision of this paper the author had the good fortune to meet David Vere-Jones, who informed him about Mandelbrot (1983) and Kagan and Vere-Jones (1988). The following is taken from Kagan and Vere-Jones (1988):

Kagan refers at several points to the suggestion of Mandelbrot (1983) that point process models for self-similar behaviour should be sought not within the class of homogeneous processes [called stationary in this paper] but within the class of processes for which the behaviour relative to a given point of the process is independent of the point selected as origin.

The authors then speculate:

One interpretation of this requirement is that the Palm distributions of the process should be the same for all points of the process.

They are on the right track because if we turn this on its head to read 'One interpretation of this requirement is that the reversed Palm distribution of the process should be stationary', then it becomes similar to defining point-stationarity by its Palm characterization (Theorem 4.1 above). Observe that (due to Theorem 4.1) the reversed Palm distribution can be generalized beyond a probability distribution and thus finite-mean Voronoi cell volume is not needed for this alternative definition.

Further, Kagan and Vere-Jones say:

What hampers us in pursuing this discussion, is that we are not aware of a well established theory for such Palm-stationary point processes. ... Any references which deal with these and related questions would be appreciated.

Further examples of Palm-stationary processes suggested by Mandelbrot, such as the Lévy dust model and zeros of Brownian motion, have a very complex point set structure, including finite accumulation points, and cannot be modelled within the standard point process framework.

The solution in the present paper of the point-stationarity problem in the case of point 
processes in $d>1$ dimensions (Definition 3.1) suggests that one might attempt to define point-stationarity in these models as follows.

Proposed definition. A random set is point-stationary if it is distributionally invariant under bijective point-shifts against any independent stationary background.

Thus our next task is to find such backgrounds and point-shifts!

\section{NOTE ADDED IN PROOF}

Conjecture 2.1 has been proved wrong by Olle Haggstrom (personal communication). It is, however, not clear at this point whether the class of bijective $\mathrm{N}^{\mathrm{o}}$-point-shifts is rich enough to define point-stationarity.

\section{Acknowledgements}

I wish to thank Francois Baccelli, Sergei Zuyev and Karl Sigman for useful discussions at an early stage of this work, the referees for scrutinizing the first hopeless version of this paper and demanding a drastic revision, and Ole Barndorff-Nielsen, Wilfrid Kendall and David Vere-Jones for helpful comments at the revision stage. This paper is dedicated to Nanna.

\section{References}

Cowan, R. (1978) The use of the ergodic theorems in random geometry. Suppl. Adv. Appl. Probab., 10, $47-57$.

Daley, D.J. and Vere-Jones, D. (1988) An Introduction to the Theory of Point Processes. New York: Springer-Verlag.

Glynn, P. and Sigman, K. (1992) Uniform Cesaro limit theorems for synchronous processes with applications to queues. Stochastic Process. Appl., 40, 29-43.

Kagan, Y.Y. and Vere-Jones, D. (1988) Statistical models of earthquake occurrence. In C.C. Heyde, Yu. V. Prohorov, R. Pyke and S.T. Rachev (eds), Athens Conference on Applied Probability and Times Series, Vol. I: Applied Probability, Lecture Notes in Statist. 114, pp. 398-425. New York: Springer-Verlag.

Mandelbrot, B.B. (1983) The Fractal Geometry of Nature. San Francisco: W.H. Freeman.

Matthes, K., Kerstan, J. and Mecke J. (1978) Infinitely Divisible Point Processes. Chichester: Wiley.

Miyazawa, M., Nieuwenhuis, G. and Sigman, K. (1999) Palm theory for random time changes. (Paper submitted.)

Nawrotzki, K. (1978) Einige Bemerkung zur Verwendung der Palmschen Verteilung in der Bedienungstheorie, Math. Operationsforschung Statist. Ser. Optim., 9 (2), 241-253.

Nieuwenhuis, G. (1994) Bridging the gap between a stationary point process and its Palm distribution. Statist. Neerlandica, 48(1), 37-62.

Nieuwenhuis, G. (1998) Ergodicity conditions and Cesaro limit results for marked point processes. Stochastic Models, 14(3), 681-714. 
Okabe, A., Boots, B. and Sugihara, K. (1992) Spatial Tessellations - Concepts and Applications of Voronoi Diagrams. New York: Wiley.

Sigman, K. (1994) Stationary Marked Point Processes: An Intuitive Approach. New York: Chapman \& Hall.

Stoyan, D., Kendall, W.S. and Mecke, J. (1987) Stochastic Geometry and Its Applications. New York: Wiley.

Thorisson, H. (1995) On time- and cycle-stationarity. Stochastic Process Appl., 55, 183-209.

Thorisson, H. (1996) Transforming random elements and shifting random fields. Ann. Probab., 24(4), $2057-2064$.

Received February 1996 and revised June 1998. 